\title{
Energy dissipation and suppression of capture cross sections in heavy ion reactions
}

\author{
D. Y. Jeung $\odot,{ }^{*}$ D. J. Hinde, E. Williams $\odot$, M. Dasgupta, E. C. Simpson, R. du Rietz $\odot,{ }^{\dagger}$ D. H. Luong, R. Rafiei $\odot,{ }^{\ddagger}$ M. Evers, ${ }^{\S}$ \\ I. P. Carter, K. Ramachandran," C. Palshetkar, D. C. Rafferty, C. Simenel $\odot$, and A. Wakhle ${ }^{\mathbb{I l}}$ \\ Department of Nuclear Physics, Research School of Physics, Australian National University, Canberra, ACT 2601, Australia
}

(Received 26 January 2021; accepted 18 February 2021; published 3 March 2021)

\begin{abstract}
Background: At energies above the capture barrier, coupled-channels (CC) calculations with a standard nuclear potential diffuseness $(0.65 \mathrm{fm})$ increasingly overestimate experimental capture cross section as the charge product $\left(Z_{1} Z_{2}\right)$ of the colliding nuclei increases. It has been suggested this may be linked to energy dissipation outside the capture barrier.

Purpose: We investigate quantitatively the role of energy dissipation in suppressing capture in heavy ion fusion reactions.

Method: The yields of sequential fission, including that resulting from deep inelastic collisions, and of fission following capture were determined simultaneously for collisions of ${ }^{18} \mathrm{O},{ }^{30} \mathrm{Si},{ }^{34} \mathrm{~S}$, and ${ }^{40} \mathrm{Ca}+{ }^{232} \mathrm{Th}$ at a range of energies around the respective capture barriers.

Results: The ratio of experimental to CC capture cross sections was found to decrease with increasing $Z_{1} Z_{2}$. Conversely, the ratio of sequential fission to capture-fission increased with increasing $Z_{1} Z_{2}$. The sum of sequential and capture fission agrees quite well with the $\mathrm{CC}$ cross sections.

Conclusions: The experimental capture fission and sequential fission cross sections, and their comparison with $\mathrm{CC}$ calculations, give a consistent picture that the increase in density overlap at the capture barrier with increasing $Z_{1} Z_{2}$ of the colliding nuclei is correlated with increasing energy dissipative processes. These compete increasingly strongly with capture as the $Z_{1} Z_{2}$ of the reaction increases. For the ${ }^{40} \mathrm{Ca}$ reaction, the total fission yield exceeds expectations from capture model calculations, indicating that deep inelastic processes occur both from trajectories that would have led to capture and also from more peripheral trajectories.
\end{abstract}

DOI: 10.1103/PhysRevC.103.034603

\section{INTRODUCTION}

Experimental measurements of capture cross sections in reactions of heavy nuclei at energies close to the capture barrier have shown that the dynamics is strongly affected by couplings to low energy collective quantum states of the colliding nuclei [1-4]. The coupled-channels (CC) model, which models the coupling between the internal states and the relative motion of the two colliding nuclei, has emerged as the most successful model of near-barrier capture [4]. However, experimental measurements of capture cross sections at energies both above [5] and far below [6,7] the barrier are consistently considerably smaller than CC capture model predictions using a standard Woods-Saxon nuclear potential

\footnotetext{
*Corresponding author: dongyun.jeung@anu.edu.au

${ }^{\dagger}$ Current address: Malmö University, Faculty of Technology and Society, Malmö 205 06, Sweden.

${ }^{\ddagger}$ Current address: University of California San Diego, 9500 Gilman Dr., La Jolla, CA 92093, USA.

${ }^{\S}$ Current address: Evoenergy, 40 Bunda Street, Canberra, ACT 2601, Australia.

"Permanent address: Nuclear Physics Division, Bhabha Atomic Research Centre, Mumbai 400085, India.

${ }^{\pi}$ Current address: ETP Ion Detect Pty Ltd. 2-8 Martha Street Clyde, NSW, Australia.
}

diffuseness of around $0.65 \mathrm{fm}$. At above-barrier energies, the discrepancies between measurements and calculations appear to increase with increasing charge product $Z_{1} Z_{2}$ of the colliding nuclei [8]. These observations suggest a deficiency in the way capture is modeled for collisions of heavy nuclei. Various approaches have been proposed to explain these reduced cross sections [9], including the possibility that deep inelastic processes may be playing a role [8].

In $\mathrm{CC}$ models, the colliding nuclei are considered to remain in a coherent superposition of their excited states until they pass inside the barrier. Capture is simulated either by imposing a boundary condition such as the incoming wave boundary condition or by use of an imaginary potential [10], at separations inside the capture barrier radius. The dissipation of kinetic energy that ultimately leads to an equilibrated compound nucleus (fusion) is thus not modeled explicitly. This treatment would mimic the physical process closely if conversion of kinetic energy into complex excitations (resulting in a highly excited "hot" composite nucleus) only occurs after passing inside the barrier. However, if processes leading to complex excitations occur (in at least some of the collisions) at separations larger than the barrier radius [11], then a lower kinetic energy at the barrier will result, leading to reduced capture cross sections. If such processes were to occur with significant probability then the method of calculating capture in the CC model would need to be modified. 
TABLE I. Details of fission and calibration (Calib.) measurements including the targets used, compound nucleus $(\mathrm{CN})$ formed, $Z_{1} Z_{2}$, and the range of energies with respect to the experimental capture barrier $\left(V_{B}\right)$ (see Table II).

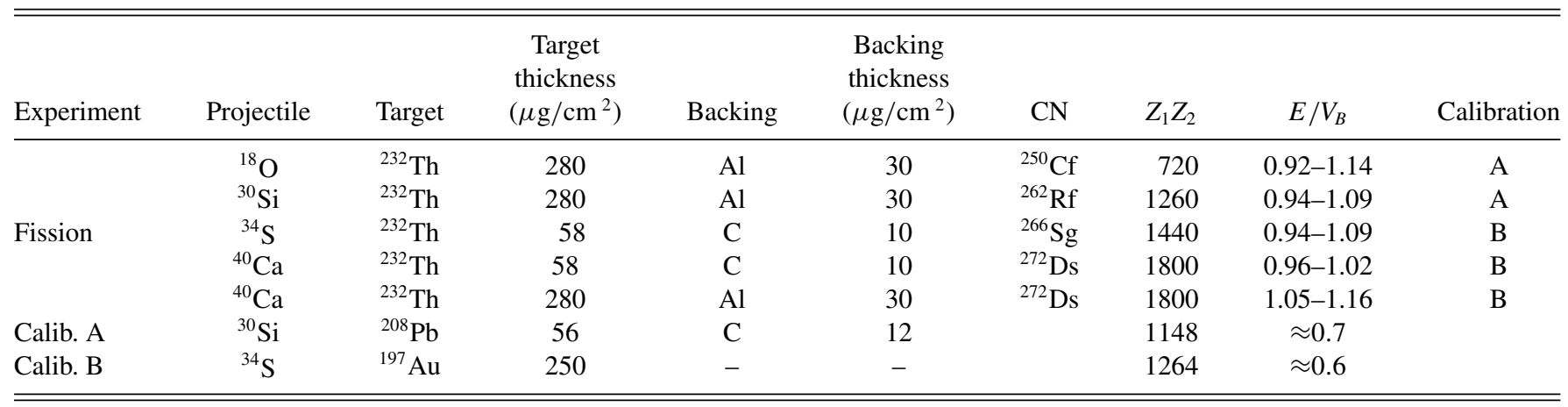

It is known that deep inelastic collisions (DICs) can result in high excitation energies in the outgoing projectile-like and/or target-like nuclei (up to hundreds of MeV) [12,13]. Although commonly associated with very heavy projectiles and high angular momentum, population of excitation energies over $10 \mathrm{MeV}$ has been observed even at sub-barrier energies $[11,14,15]$, typically associated with the transfer of several nucleons (multinucleon transfer). These measurements do not distinguish between excitation of the lighter (projectile-like) nucleus and the heavier (target-like) nucleus. For the kinetic energy to be converted from relative motion essentially irreversibly (classical energy dissipation), a high level density should be present, where multiple couplings between overlapping states should prevent reversible coupling back to relative motion [16]. This is more likely to be the case for excitation of a heavy (target-like) nucleus because of the much higher level density at a given excitation energy. Thus, to make a quantitative study of energy dissipation and fusion, a signal of energy dissipation in the target-like nucleus is desirable.

The observation of fission following a nuclear collision is a signature that kinetic energy has been converted to internal energy, and is thus an indication of essentially irreversible energy dissipation. This work exploits fission of the compound nucleus $(\mathrm{CN})$ and of excited heavy target-like nuclei produced in dissipative collisions to study the interplay between these processes in a quantitative way. Measurements have been made as a function of $Z_{1} Z_{2}$ and beam energy, from below to above barrier. The fissile target nucleus ${ }^{232} \mathrm{Th}$ was used since low fission barriers $(\approx 6 \mathrm{MeV})$ [17] results in fission not only following capture, but also following (multi)nucleon transfer processes that lead to excitation energies higher than the fission barrier.

A few complexities arise in identifying capture cross sections in reactions of heavy nuclei. Unlike relatively light systems (e.g., ${ }^{16} \mathrm{O}+{ }^{144} \mathrm{Sm}$ ), capture inside the Coulomb barrier does not necessarily lead to fusion. Following capture, heavy systems may proceed to form a compact $\mathrm{CN}$ or can break apart prior to full shape equilibration, in a process called quasifission (QF). Quasifission increasingly competes with $\mathrm{CN}$ formation with increasing charge product of the colliding nuclei [18]. Since this work investigates the link between energy dissipative processes that occur outside the barrier and those that pass inside the barrier (capture), the distinct processes of interest are capture (leading to both fusion-fission and quasifission) and sequential fission of the target-like nucleus after energy dissipative reactions. Hence, in the rest of this paper the term capture rather than fusion is used. Thus the observed fission consists of (i) fusion-fission, (ii) quasifission, and (iii) fission of target-like nuclei (fission following transfer or deep inelastic reactions, here referred to collectively as sequential fission).

\section{EXPERIMENTS}

Experiments were carried out with projectiles of ${ }^{18} \mathrm{O},{ }^{30} \mathrm{Si}$, ${ }^{34} \mathrm{~S}$, and ${ }^{40} \mathrm{Ca}$, which bombarded targets of ${ }^{232} \mathrm{Th}$ at energies from below to above the respective capture barriers. Beams were accelerated using the 14UD tandem accelerator at the Australian National University Heavy Ion Accelerator Facility. The superconducting linear post accelerator was used to provide the higher energy beams of ${ }^{40} \mathrm{Ca}$. Pulsed beams with a typical width of 1-1.5 ns FWHM and pulse separation of $106.7 \mathrm{~ns}$ were incident on thin ${ }^{232} \mathrm{Th}$ films deposited on either $\mathrm{C}$ or Al backings. Table I shows details of the systems studied in this work, giving information on all reactions and calibration runs including information on targets, backings, and energy ranges.

Binary reaction products including pairs of fission fragments, and scattered beam particles and recoils, were detected in coincidence in the CUBE spectrometer [18], consisting in this work of two large area position-sensitive multiwire proportional counters (MWPCs). The two MWPCs were placed $180 \mathrm{~mm}$ away from the target, whose normal was oriented at $60^{\circ}$ to the beam axis. This minimized energy loss of the fission fragments in the targets, and avoided shadowing of the detectors by the target frame. The back angle counter was centered at $90^{\circ}$, giving angular coverage of $55^{\circ}$ to $130^{\circ}$. The front angle counter was centered at $45^{\circ}$, covering $5^{\circ}$ to $80^{\circ}$.

The wire grids in each MWPC, connected to delay lines, allow position to be determined for each detected particle with $1 \mathrm{~mm}$ accuracy. The time difference between the delayed readouts from opposite ends gives the position coordinate $(X, Y)$ on the active area of the detector. The cathode signal provided time-of-flight (ToF) information (measured with respect to the beam reference RF signal) and also the energy loss of the fission fragments. Using the information of position and ToF, the velocity vectors of the fission fragments were reconstructed event by event, allowing identification of the type of fission 

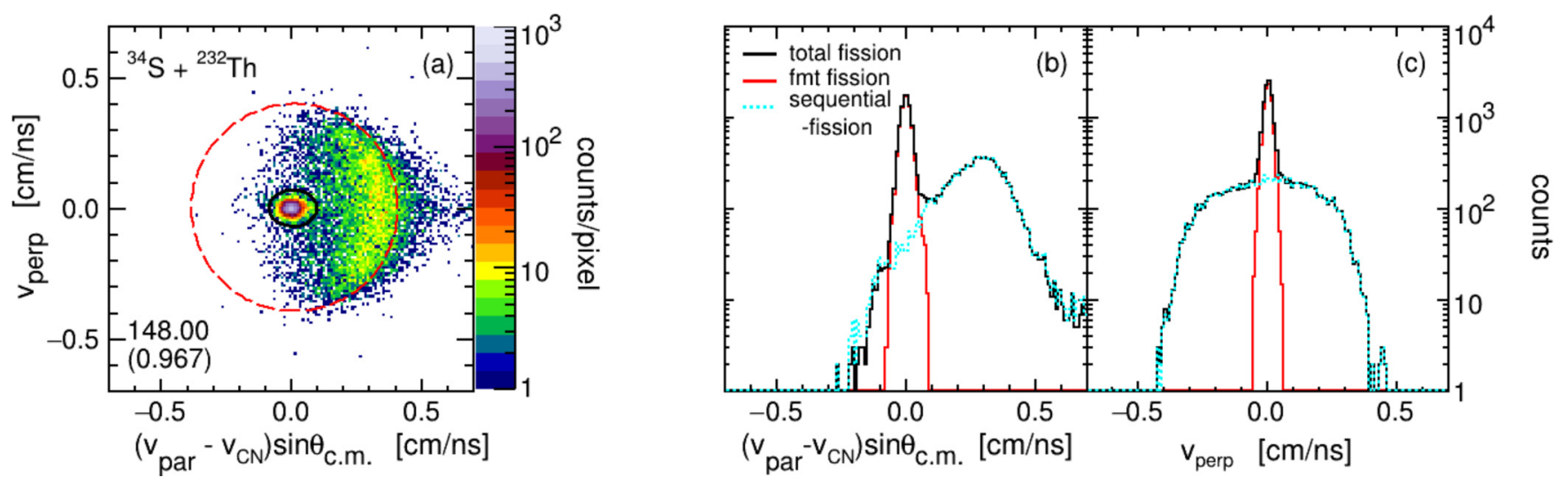

FIG. 1. Panel (a) is an example of the experimental source velocity distribution for ${ }^{34} \mathrm{~S}+{ }^{232} \mathrm{Th}$ at $E_{\mathrm{c} . \mathrm{m} .}=148.0 \mathrm{MeV}\left(E / V_{B}=0.967\right)$. FMT fission events are populated in the center of the plot and events outside of a FMT fission boundary (black ellipse) are dominated by sequential fission events. The dashed red circle is the expected boundary of those events. Panels (b) and (c) shows projections of (a) onto $\left(v_{\text {par }}-v_{\mathrm{CN}}\right) \sin \theta_{\text {c.m. }}$ and $v_{\text {perp }}$, respectively.

event through their different kinematical characteristics, as described in Ref. [18].

As well as the MWPCs, two ion implanted $\mathrm{Si}$ detectors (monitors), were mounted symmetrically about the beam axis in the vertical plane at angles of $30^{\circ}$ to detect elastically scattered particles for absolute cross section determination relative to Rutherford scattering. Two calibration measurements of elastic scattering (for the ${ }^{30} \mathrm{Si}+{ }^{208} \mathrm{~Pb}$ and ${ }^{34} \mathrm{~S}+{ }^{197} \mathrm{Au}$ reactions) were carried out at energies far below their barriers.

\section{DATA ANALYSIS AND INTERPRETATION}

The goal of this work is to determine the competition between capture and dissipative (transfer and deep) inelastic processes both qualitatively and quantitatively as a function of $Z_{1} Z_{2}$. In order to achieve this goal, two quantities needed to be determined.

The first was to determine the cross sections of those fission events where all the momentum of the projectile was transferred to the composite system. This is referred to as full momentum transfer (FMT) fission, and is associated with capture of the projectile, followed by fusion-fission or $\mathrm{QF}$ [19-21].

The second was to determine the ratio of FMT fission to the total fission events, made up of FMT fission and those fission events with a third (projectile-like) nucleus being ejected. From the ratio, total and sequential fission cross sections were estimated. This sequential fission follows an initial transfer or deep inelastic reaction [20].

\section{A. Source velocities of fission events}

From the deduced laboratory velocity vectors of fission fragments, two components of the velocity vector of the nucleus undergoing fission can be determined. As described in Refs. [20,22], momentum conservation in the center-of-mass frame of the fission event allows the source velocity along the beam axis $\left(v_{\text {par }}\right)$ and perpendicular to both the beam axis and the fission axis ( $\left.v_{\text {perp }}\right)$ to be determined.

For FMT fission at energies not too far above the capture barrier, the value of $v_{\text {par }}$ should be the center-of-mass velocity, or equivalently the expected velocity of the $\mathrm{CN}$ following fusion $\left(v_{\mathrm{CN}}\right)$, and $v_{\text {perp }}$ should be zero. It was recently shown [22] that the distribution of events around $\left(v_{\mathrm{par}}-v_{\mathrm{CN}}\right)=0$ becomes broader as $\theta_{\text {c.m. }}$. moves further from $90^{\circ}$, due to a trigonometrical effect. This can simply be corrected by scaling the deviation of $\left(v_{\mathrm{par}}-v_{\mathrm{CN}}\right)$ from zero by $\sin \theta_{\mathrm{c} . \mathrm{m} .}$. The deduced fission source velocity is therefore plotted as $\left(v_{\text {par }}-\right.$ $\left.v_{\mathrm{CN}}\right) \sin \theta_{\text {c.m. }}$ vs $v_{\text {perp }}$. FMT capture-fission events should thus lie close to $(0,0)$, at the center of the distribution, for all angles.

A typical source velocity distribution for ${ }^{34} \mathrm{~S}+{ }^{232} \mathrm{Th}$ at $E_{\text {c.m. }}=148.0 \mathrm{MeV}$ is shown Fig. 1 . The intense group peaked at $(0,0)$ corresponds to FMT fission events. These events show a distribution in both $\left(v_{\text {par }}-v_{\mathrm{CN}}\right) \sin \theta_{\text {c.m. }}$. and $v_{\text {perp }}$. This is expected, due to the evaporation of light particles (predominantly neutrons, but also protons and $\alpha$ particles) from the system either before or after scission, perturbing the fission fragment velocity vectors. Being emitted almost isotropically, the mean of the distribution is not affected. The width of the measured FMT fission source velocity distribution arises mainly from this physical evaporation process, rather than the effects of instrumental resolution or angular straggling in the target.

In contrast to the narrow distribution for FMT fission events, sequential fission events have a much wider spread in source velocities shown in Figs. 1(b) and 1(c). This arises because of the much larger recoil momentum of the ejected beam-like particle compared with evaporated neutrons. For simplicity, we initially suppose fission could be initiated by a recoiling projectile-like nucleus having the momentum of the beam particle in the center-of-mass frame. The recoil would have equal and opposite momentum to the beam-like particle, independent of angle. The recoil velocity distribution lies on a circle of radius $v_{\mathrm{CN}}$. This circle (red dashed line) is drawn in Fig. 1(a). 


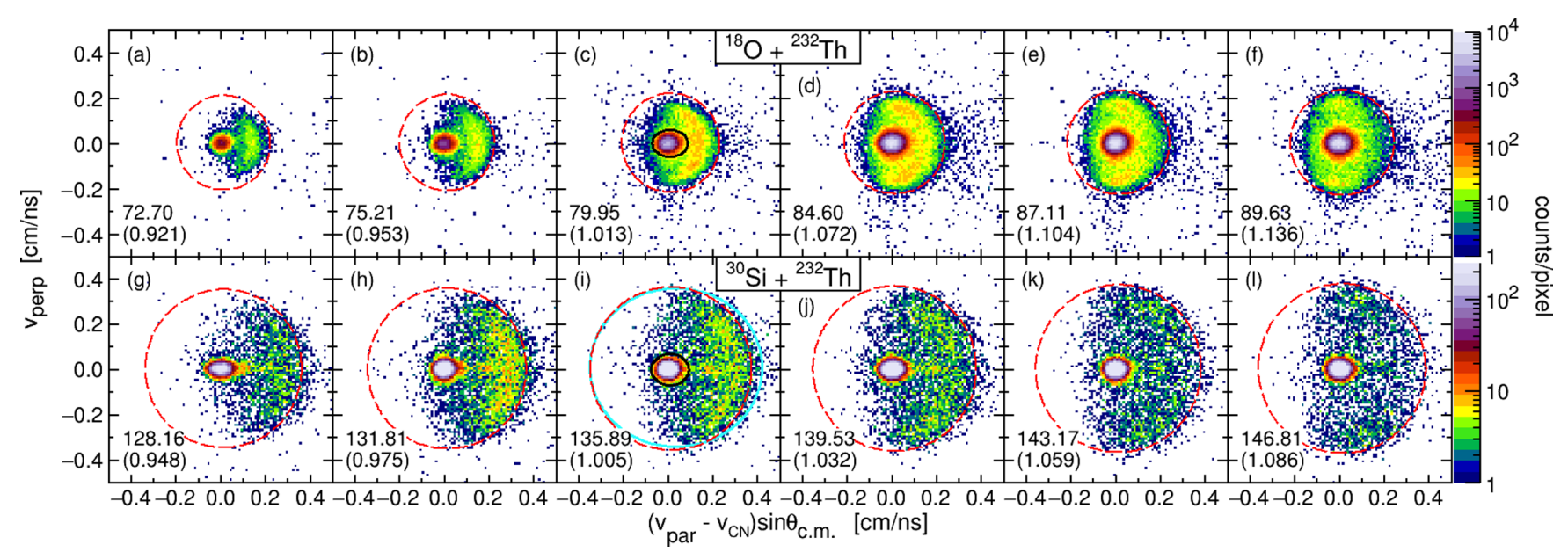

FIG. 2. Experimentally deduced distributions of the source velocity components (see text) of the fissioning nuclei, $\left(v_{\text {par }}-v_{\mathrm{CN}}\right) \sin \theta_{\text {c.m. }}$.

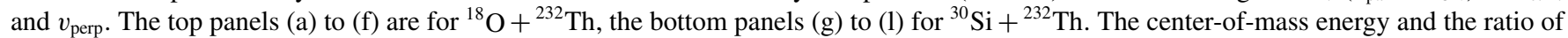
the beam energy to the capture barrier $\left(E / V_{B}\right)$ are given in each panel. The small black elliptical gates in (c) and (i) are examples of the gates selecting FMT fission events. Sequential fission events should be generally constrained inside a circle radius of $v_{\mathrm{CN}}$ (red circle) but could also be outside this limit [cyan ellipse in panel (i)], as described in the text.

Sequential fission is expected for peripheral reactions on trajectories near the grazing angle. At energies near the capture barrier $V_{B}$, the grazing angle is backwards of $90^{\circ}$ at $E \sim V_{B}$. The recoil velocity then has a value of $v_{\mathrm{par}}>v_{\mathrm{CN}}$, and $v_{\text {perp }} \neq 0$. At $E>V_{B}$, the grazing angle moves through $90^{\circ}$, then at $E \gg V_{B}$ to forward angles, where $v_{\mathrm{par}}<v_{\mathrm{CN}}$. This is reflected in the dependence of the experimental distributions of source velocity vectors shown in Figs. 2 and 3.

Because of the change in the Coulomb field, transfer reactions leading to excitation energies higher than the fission barrier are usually charged-particle stripping reactions. Thus the recoil momentum is less than that of the projectile, and, for each individual transfer channel, the events will lie on the surface of a sphere with radius less than $v_{\mathrm{CN}}$. The source velocity distribution of the sequential fission events thus shows a diffuse distribution, which in general lies inside the kinematical boundary for elastic scattering, a circle of radius $v_{\mathrm{CN}}$ centered at $(0,0)$. This condition is shown by the red dashed circles in Figs. 2 and 3.

With increasing projectile momentum, the radius of the sequential fission source velocity distributions increases due to the larger recoil velocity of the target-like nucleus. For heavy projectiles like ${ }^{40} \mathrm{Ca}$, sequential fission of a heavy target-like nucleus formed after very mass-asymmetric $\mathrm{QF}$ events could also occur. Very asymmetric QF events may result in a projectile-like nucleus which is heavier than the initial projectile, and has more momentum. If the complementary target-like nucleus undergoes fission, then this event can lie outside the boundary. According to Ref. [23], the fraction of such fission events is small, and thus the dominant origin of sequential fission in this work should be fission following transfer and deep inelastic reactions.

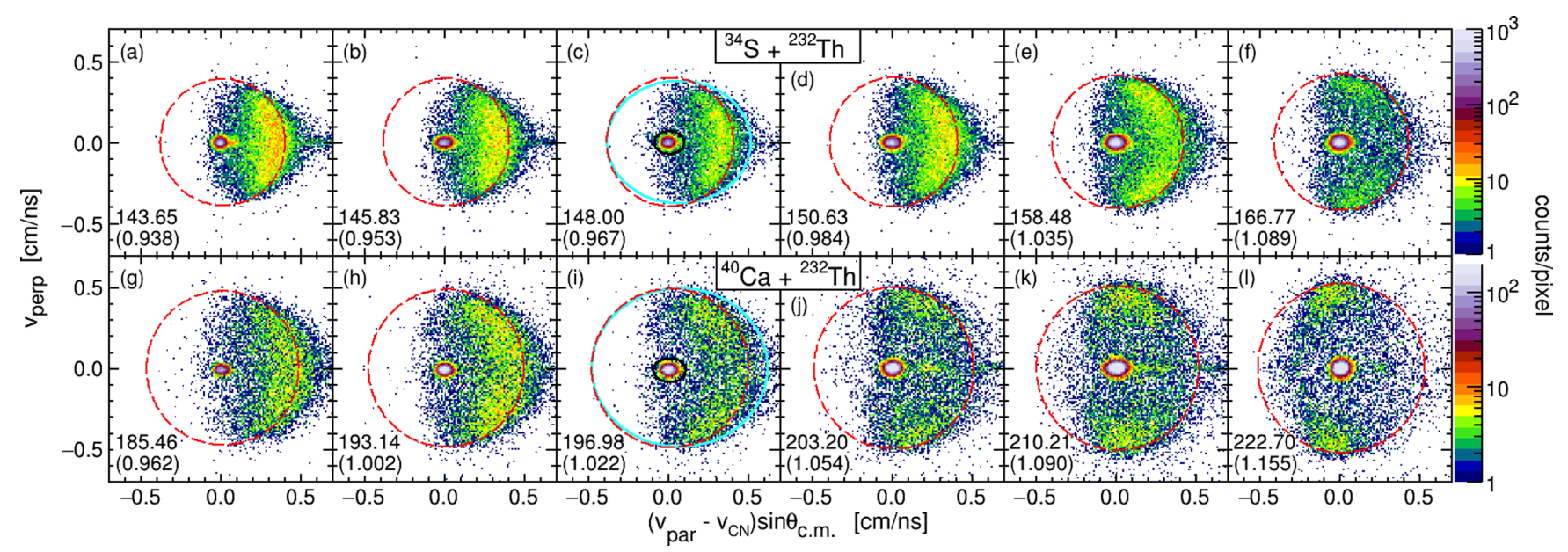

FIG. 3. Experimentally deduced distributions of fission source velocity components $\left(v_{\text {par }}-v_{\mathrm{CN}}\right) \sin \theta_{\text {c.m. }}$ and $v_{\text {perp }}$. The top panels (a) to (f) are for ${ }^{34} \mathrm{~S}+{ }^{232} \mathrm{Th}$, the bottom panels (g) to (l) are for ${ }^{40} \mathrm{Ca}+{ }^{232} \mathrm{Th}$. The concentration of events at $(0.6,0.0)$ arises from reactions with light target impurities. Note the change in the velocity scale for these heavier projectile nuclei compared to those in Fig. 2. 


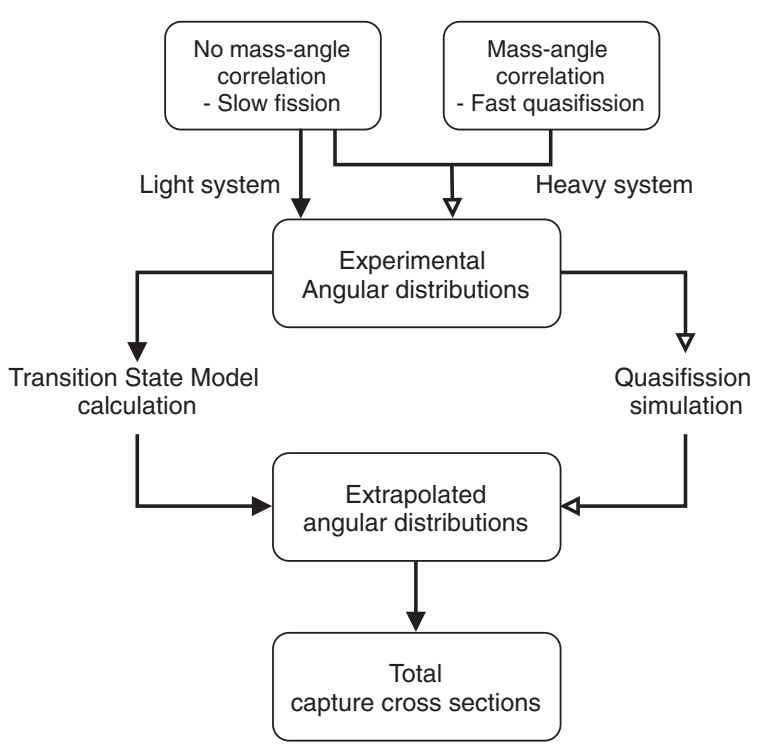

FIG. 4. Flow chart of capture cross section determination depending on the category of the system, light or heavy, classified by the presence of a mass-angle correlation in the mass-angle distribution (MAD).

\section{B. Full momentum transfer fission cross sections}

For reactions of heavy ions with ${ }^{232} \mathrm{Th}$, the capture cross sections $\left(\sigma_{\text {cap }}\right)$ can be taken as the FMT fission cross sections since cross sections for evaporation residues are very small in comparison.

Depending on the $Z_{1} Z_{2}, \sigma_{\text {cap }}$ consists of predominantly fusion-fission or a mixture of fusion-fission and QF. It is important to identify the presence of QF in the FMT fission events because in this work it was found necessary to use different methods to obtain $\sigma_{\text {cap }}$ from the fission angular distributions, depending on the prevalence of fast quasifission, as described below. The sequence of steps in determining the experimental FMT fission cross sections is shown in Fig. 4.

\section{Characteristics of fast quasifission}

FMT fission can include both fusion-fission and QF. According to Ref. [24], the onset of QF was predicted when $Z_{1} Z_{2}>1600$, but recent studies and observations $[18,25,26]$, including this work, have observed $\mathrm{QF}$ for $Z_{1} Z_{2}$ much less than 1600 . QF competes increasingly with true fusion (formation of a compact $\mathrm{CN}$ ) as the $Z_{1} Z_{2}$ of the reaction partners, or the mean fissility [18] become larger. Therefore, the different physics controlling the angular distributions of fusion-fission and QF needs to be taken into account in determining capture cross sections [5,18,20,27,28] from measured angular distributions. This proved important for the ${ }^{34} \mathrm{~S}+{ }^{232} \mathrm{Th}$ and ${ }^{40} \mathrm{Ca}+{ }^{232} \mathrm{Th}$ reactions investigated in this work.

Quasifission shows some characteristics that are similar to fusion-fission. These include damped kinetic energies [23] and fragment masses typically being far from those in the entrance channel. The presence of fast QF following capture reactions has been seen in the mass (or mass ratio) distributions whether measured at one angle or integrated over a wide range of angles [27]. However, the presence of fast QF can be seen most clearly in the correlation of mass with angle in experimental mass-angle distributions (MADs). This correlation can have significant implications for the determination of fission cross sections, as described below.

MADs show the fission mass or mass-ratio $M_{R}$ [see Eq. (1)] as a function of the scattering angle $\left(\theta_{\text {c.m. }}\right)$. The mass ratio $\left(M_{R}\right)$ of any binary event, including FMT fission and quasielastic scattering, was derived in this work using the experimental center-of-mass velocities $v_{\text {c.m.1 }}$ and $v_{\text {c.m.2 }}$ of the two fragments. Based on conservation of linear momentum $[20,23], M_{R}$ is given by

$$
M_{R}=\frac{v_{\text {c.m. } 2}}{v_{\text {c.m. } 1}+v_{\text {c.m. } 2}}=\frac{M_{1}}{M_{1}+M_{2}},
$$

where $M_{1}$ and $M_{2}$ are the masses of the two fragments.

Figure 5 shows examples of experimental FMT mass-angle distributions for each reaction investigated in this work. FMT (binary) events were selected by applying a gate centered around $(0,0)$ in the source velocity distributions, as shown by the black ellipses in Figs. 2 and 3, panels (c) and (i). The MADs display a single fission event as a pair of points representing the two fission fragments, mirrored about $M_{R}=0.5$ and $\theta_{\text {c.m. }}=90^{\circ}$ [18]. Thus one fragment is at $\left(M_{R}, \theta_{\text {c.m. }}\right)$ and the complementary fragment is at $\left(1-M_{R}, 180^{\circ}-\theta_{\text {c.m. }}\right)$.

Depending on the distribution of lifetimes of the rotating nuclear systems, a correlation between fission fragment masses and emission angles may be observed in the MAD, as sketched in Fig. 5(a). The fast QF process [18] shows a strong mass-angle correlation since a short contact time allows only a limited amount of mass exchange and small rotation angle. As the contact time gets longer, more mass flow can occur between the two constituents, and eventually the system reaches the (lowest energy) mass-symmetric configuration. Therefore, the presence of a mass-angle correlation is a signature of fast $\mathrm{QF}$, and provides quantitative information of QF timescales between $10^{-21}$ and $10^{-20} \mathrm{~s}$ [29].

Apart from the MADs shown in Fig. 5, the full range of experimental MADs for the reactions investigated in this work are presented in Refs. $[18,28,30]$ and, for ${ }^{40} \mathrm{Ca}+{ }^{232} \mathrm{Th}$, in the Appendix of this work. Based on the observation of mass-angle correlations and thus the contribution from fast $\mathrm{QF}$, the systems are classified as light or heavy. ${ }^{18} \mathrm{O}+{ }^{232} \mathrm{Th}$ and ${ }^{30} \mathrm{Si}+{ }^{232} \mathrm{Th}$ are classified as light systems. The MADs for ${ }^{18} \mathrm{O}+{ }^{232} \mathrm{Th}$ shows symmetric mass-distributions peaked at $M_{R}=0.5$ [Fig. 5(b)] and angular distributions symmetric about $\theta_{\text {c.m. }}=90^{\circ}$ for all masses and beam energies. ${ }^{30} \mathrm{Si}+{ }^{232} \mathrm{Th}$ includes a small fraction [30] of fast $\mathrm{QF}$ events [Fig. 5(c)] but in general shows mass distributions peaked at mass symmetry. However, both the ${ }^{34} \mathrm{~S}+{ }^{232} \mathrm{Th}$ and ${ }^{40} \mathrm{Ca}+{ }^{232} \mathrm{Th}$ reactions show strong mass-angle correlations and have significant mass-asymmetric components for all energies [Figs. 5(d) and 5(e)]. These reactions were classified as heavy systems.

In order to obtain total FMT fission cross sections, the fission angular distributions must be extrapolated to angles beyond the MWPC detector coverage. Depending on the fraction and characteristics of the QF component of the fission events, 


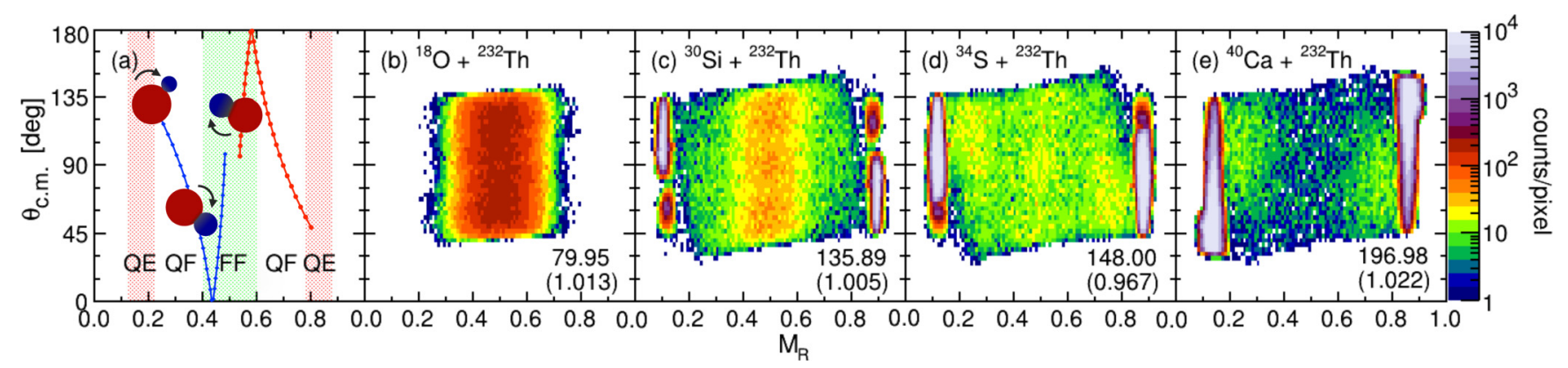

FIG. 5. Panel (a) shows the regions corresponding to different reaction process depending on the sticking time of the dinuclear system. These are quasielastic scattering (QE), quasifission (QF), and fusion-fission (FF). Adapted from Ref. [18]. Panels (b) to (e) show the mass-angle distributions generated by applying a gate on the full momentum transfer (FMT) fission, such as the black elliptical gates in panels (c) and (i) of Figs. 2 and 3.

different methods were used to achieve this for the light and heavy systems, as described below.

\section{Angular distributions for slow fission processes}

Because the ${ }^{18} \mathrm{O}+{ }^{232} \mathrm{Th}$ and ${ }^{30} \mathrm{Si}+{ }^{232} \mathrm{Th}$ reactions show little or no evidence for a mass-angle correlation, the FMT fission angular distributions should be well described with the transition state model (TSM) [31,32], using quantum numbers, the total angular momentum $J$, the projection of $J$ on the nuclear symmetry (fission) axis $K$, and the projection of the space-fixed axis $M$. Fitting the measured angular distributions was achieved by optimizing the value of $K_{0}$, the variance of the $K$ distribution, taking the $J$ distribution for capture to be that calculated with the coupled-channels model at each energy. The measured and fitted angular distributions for the ${ }^{30} \mathrm{Si}+{ }^{232} \mathrm{Th}$ measurements are shown in Fig. 6. The total fission cross sections were obtained by integrating the fitted angular distributions multiplied by $2 \pi \sin \theta_{\text {c.m. }}$, over angles from $90^{\circ}$ to $180^{\circ}$.

\section{Angular distributions for fast quasifission}

Measured mass angle distributions for reactions of heavy projectiles on deformed actinide nuclei show that QF fragments with masses closer to the projectile than to mass symmetry are found in a relatively narrow range of angles, with the target-like fragments at the complementary angle. This is seen clearly in Figs. 5(c) and 5(d). The total angular distributions of all fragments from fast QF thus show behavior that cannot be fitted with the TSM formalism [14,33,34], since the required condition that the system has lost all "memory" of the projectile mass and direction is not met. Therefore, fitting angular distributions with the TSM may give the wrong cross sections if the reactions have a significant fraction of fast QF.

In this work, to fit the measured QF angular distributions and extrapolate the cross sections to angles outside the measured range, an empirical classical QF model was used. This is based on the phenomenological approach of Refs. [23,25]. Using the Monte Carlo technique [18,28,29], the goal is to reproduce experimental MADs (and thus the projectile-like and target-like components of the angular distribution) by optimizing the distribution of sticking times for the reaction (see the Appendix).
Results of this fitting procedure are shown in Fig. 7, for two contrasting cases: ${ }^{34} \mathrm{~S}+{ }^{232} \mathrm{Th}$ at a sub-barrier energy and ${ }^{40} \mathrm{Ca}+{ }^{232} \mathrm{Th}$ at a c.m. energy well above the capture barrier. Panels (a)-(c) show respectively the experimental MAD, the simulated MAD, and the angular distributions for ${ }^{34} \mathrm{~S}+{ }^{232} \mathrm{Th}$. Panels (d)-(f) show the same for ${ }^{40} \mathrm{Ca}+{ }^{232} \mathrm{Th}$. Full details of the generation of the simulated MADs for ${ }^{34} \mathrm{~S}+{ }^{232} \mathrm{Th}$ are

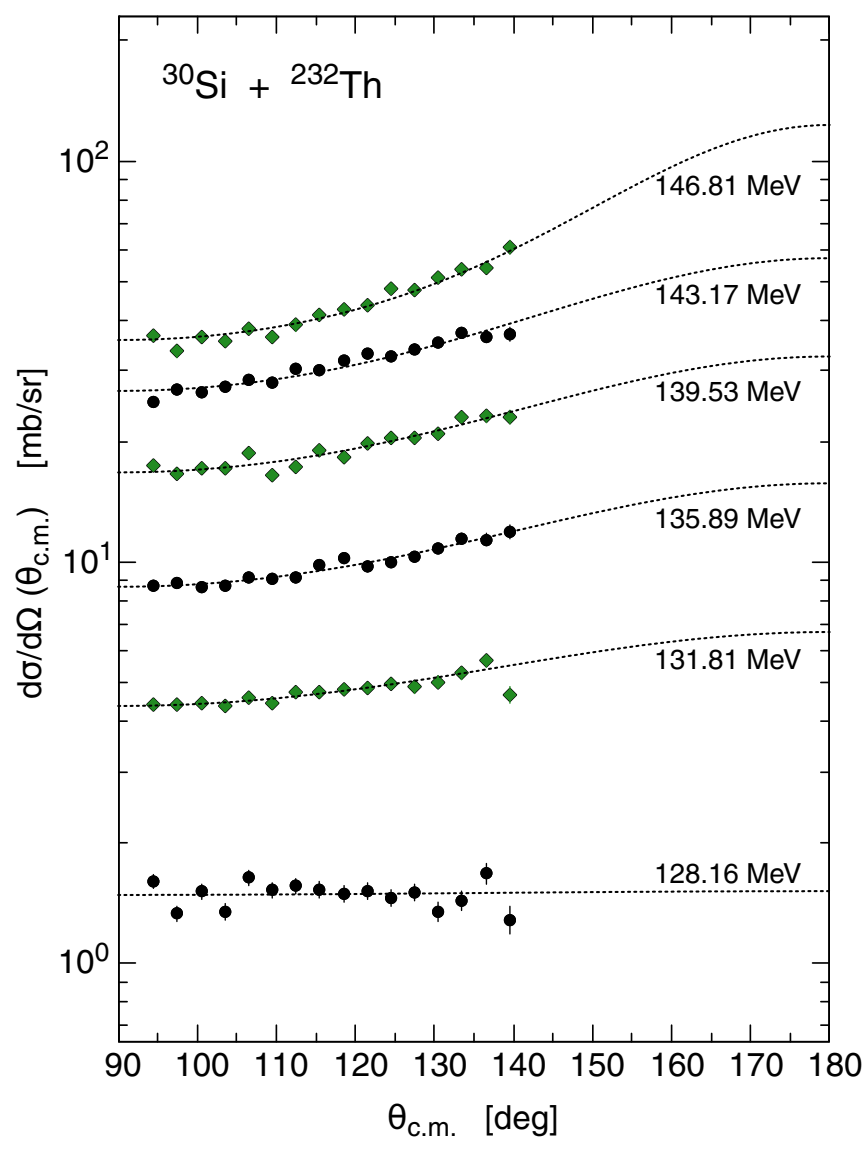

FIG. 6. The fission fragment angular distribution for ${ }^{30} \mathrm{Si}+{ }^{232} \mathrm{Th}$ at different center-of-mass energies as a function of the scattering angle in the center-of-mass frame. The lines are the results of the TSM calculations fit to the experimental distribution and thus extrapolations to $180^{\circ}$. 

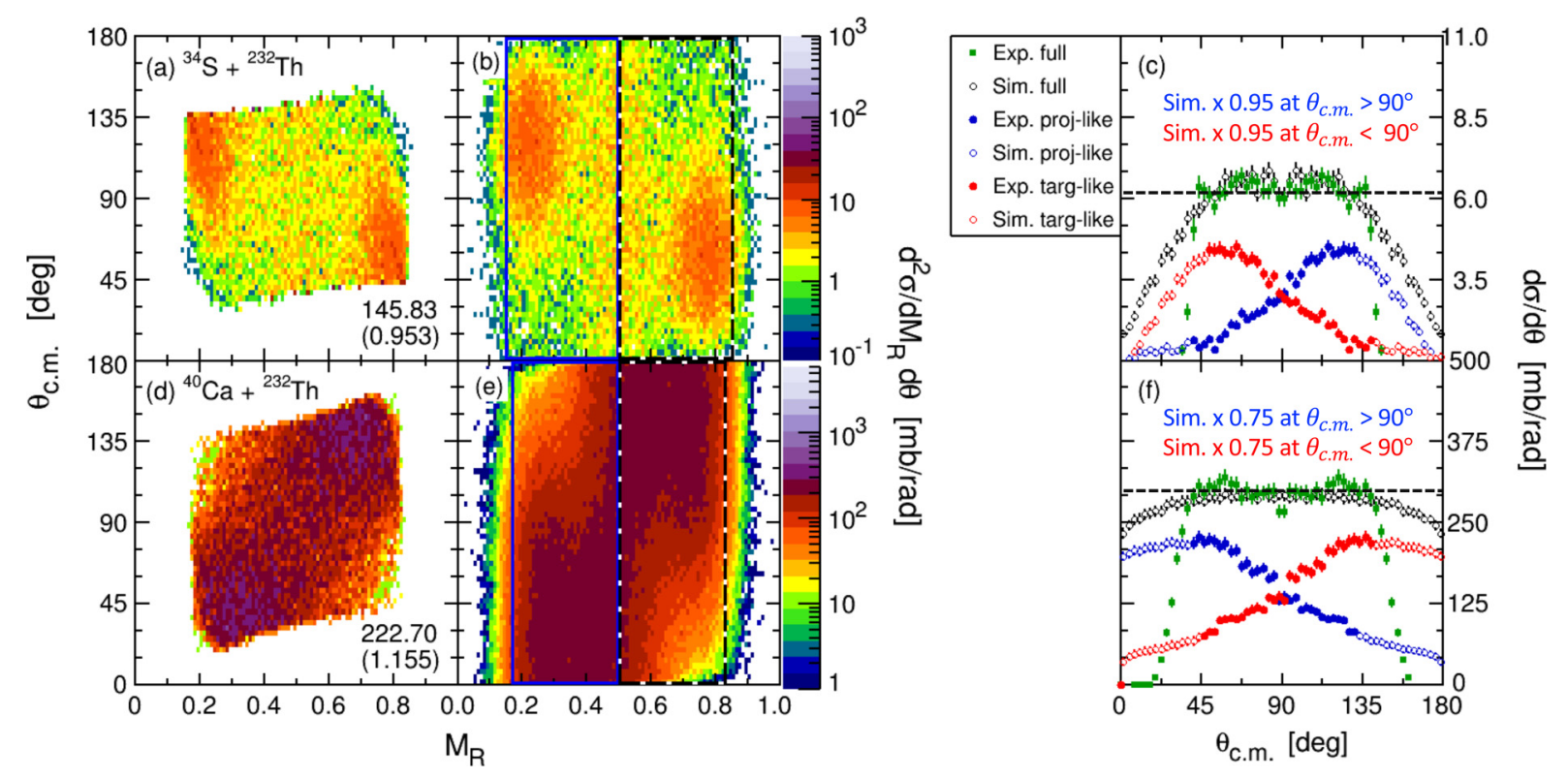

FIG. 7. (a) and (d) show experimental MADs, (b) and (e) show simulated MADs for ${ }^{34} \mathrm{~S}+{ }^{232} \mathrm{Th}$ and ${ }^{40} \mathrm{Ca}+{ }^{232} \mathrm{Th}$ respectively. Fission angular distributions are obtained by projecting distributions onto the $y$ axis $\left(\theta_{\text {c.m. }}\right)$ shown in (c) and (f). Blue and black boxes are restricted ranges to produce an angular distribution of the projectile-like fission (red data, $M_{R}<0.5$ ) and target-like fission (blue data, $M_{R}>0.5$ ) where solid circles are from experiments and hollow circles from simulations. Green square and black hollow points are the distributions for the full range of $M_{R}$.

given in Ref. [28], while for ${ }^{40} \mathrm{Ca}+{ }^{232} \mathrm{Th}$ details are provided in the Appendix of this work.

Over the experimental angular range [as seen in Figs. 7(a) and $7(\mathrm{~d})$ ], the simulated MADs successfully reproduce the measurements. For ${ }^{34} \mathrm{~S}+{ }^{232} \mathrm{Th}$ the peak in yield of projectilelike fast QF fragments is at backward angles because the angular momentum is low. For ${ }^{40} \mathrm{Ca}+{ }^{232} \mathrm{Th}$ the angular momentum is high, and correspondingly the peak is at forward angles.

Projection of the full experimental MAD (which displays all fission fragments [18]) onto the $y$ axis gives the total fission fragment angular distribution. This is shown by the green square points in Figs. 7(c) and 7(f). The distributions for the two systems look very similar, and appear to be well fitted by $d \sigma / d \theta=$ constant (corresponding to $d \sigma / d \Omega \propto 1 / \sin \theta$ ) as shown by the dashed lines.

As noted above, the projectile-like $\left(M_{R}<0.5\right)$ and targetlike $\left(M_{R}>0.5\right)$ fragment angular distributions peak in opposite directions for the two reactions. This is clearly shown by the full blue and red circles respectively in Figs. 7(c) and 7(f). Most importantly for determining the fission cross sections, the simulations predict very different extrapolations to $0^{\circ}$ and $180^{\circ}$ for the two reactions. The simulated angular distributions outside the angular coverage of the detector system are given by the hollow blue and red points.

Summing the projectile-like and target-like simulations gives the total simulated fission fragment angular distributions, which are indicated by the hollow black circles. Naturally the extrapolations of the total $d \sigma / d \theta$ to $0^{\circ}$ and $180^{\circ}$ are quite different for the two reactions. For ${ }^{34} \mathrm{~S}+{ }^{232} \mathrm{Th}$, at a lower $\mathrm{E} / \mathrm{V}_{B}$, the total fission cross section from the simulation is $20 \%$ smaller than the commonly made assumption that $d \sigma / d \theta=$ constant, showing the level of ex- perimental uncertainty that can arise from this simplifying assumption.

The total cross sections for FMT fission events were obtained by integrating the projectile-like $\left(M_{R} \leqslant 0.5\right)$ angular distributions (the target-like angular distributions of course give the same result) over the full angular range from $0^{\circ}$ to $180^{\circ}$, using the experimental data where the detectors provided coverage, and using the simulations matched to experiment for angles closer to $0^{\circ}$ and $180^{\circ}$.

The projectile-like $\left(M_{R} \leqslant 0.5\right)$ fission angular distributions for the ${ }^{34} \mathrm{~S}+{ }^{232} \mathrm{Th}$ and ${ }^{40} \mathrm{Ca}+{ }^{232} \mathrm{Th}$ reactions that were used to determine the total FMT fission cross sections are shown in Fig. 8. Depending on beam energy, especially where fast QF products are dominant in the MAD, the simulations required rescaling (as indicated) in order to make a smooth transition between the experimental data and simulated data. The simulations were focused on matching the experimental data where the QF yield was largest, at either forward angles or backward angles depending on beam energy (see Fig. 17 for ${ }^{40} \mathrm{Ca}+{ }^{232} \mathrm{Th}$ ). It seems likely that there should be a correlation between the sticking time and angular momentum [35], but this has not yet been experimentally quantified, and so is not included in the simulation code. It is thought that this is the reason for the required scaling of the simulation in some cases.

\section{Experimental capture barrier energies}

The experimental capture barrier energies for each reaction were obtained by fitting the capture cross sections when presented as a function of $1 / E_{\text {c.m. }}$, as shown in Fig. 9. The inverse of the $x$-axis intercept of a linear fit to the experimental data at above barrier energies gives the experimental mean barrier energy. This is based on a classical picture of passage over 

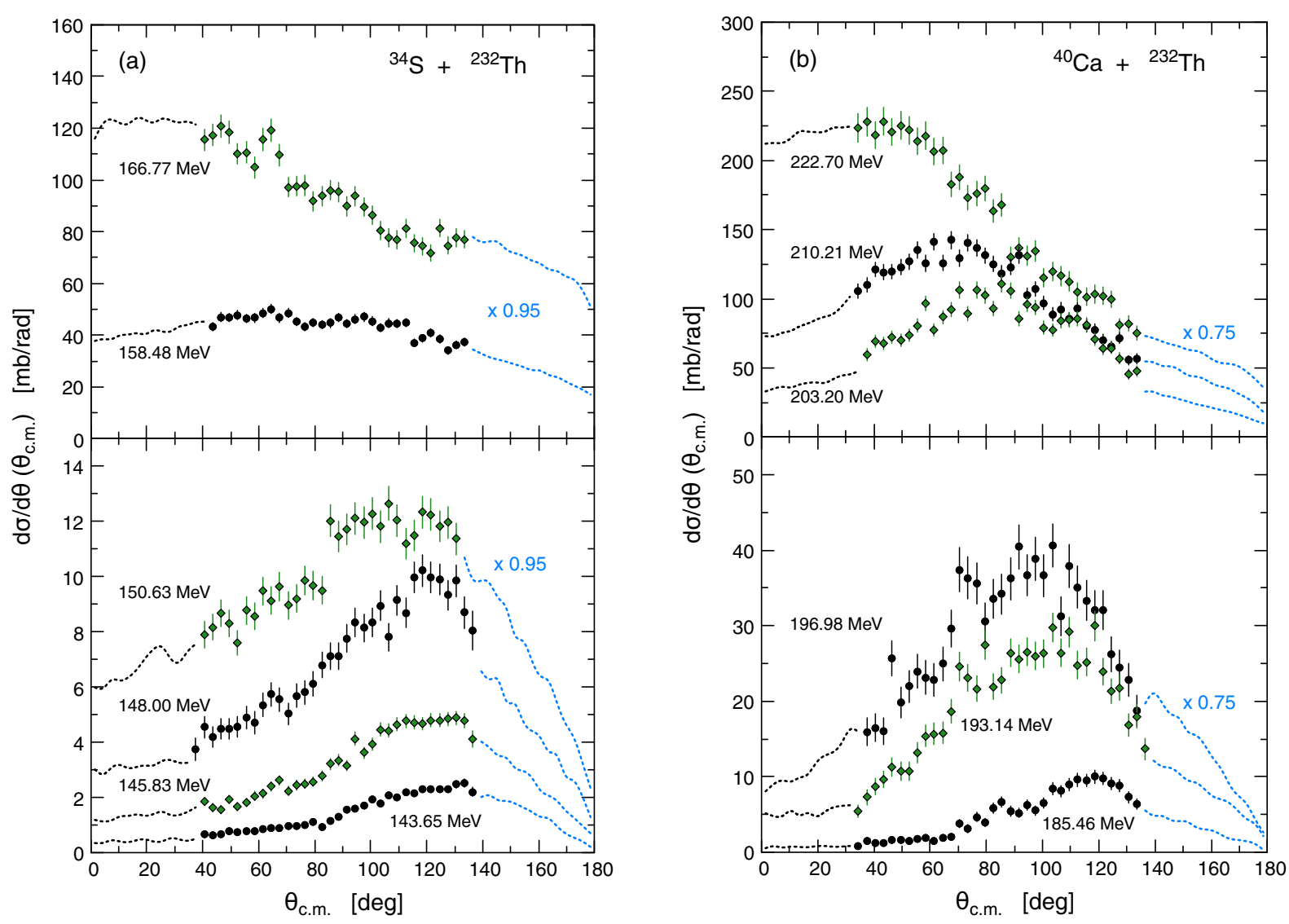

FIG. 8. Fission fragment angular distributions $\left(M_{R}<0.5\right)$ for ${ }^{34} \mathrm{~S}+{ }^{232} \mathrm{Th}$ (a) and ${ }^{40} \mathrm{Ca}+{ }^{232} \mathrm{Th}$ (b) at different c.m. energies as indicated. $d \sigma / d \theta$ is shown as a function of the scattering angle in the center-of-mass frame. Filled points are from the experiment and lines are from $\mathrm{QF}$ simulations. Extrapolated angular distributions at backward angles from $90^{\circ}$ to $180^{\circ}$ were scaled to match the experiment by factors indicated by the labels (see text).

a single barrier whose radius is independent of angular momentum. This method is reasonably accurate for cross section

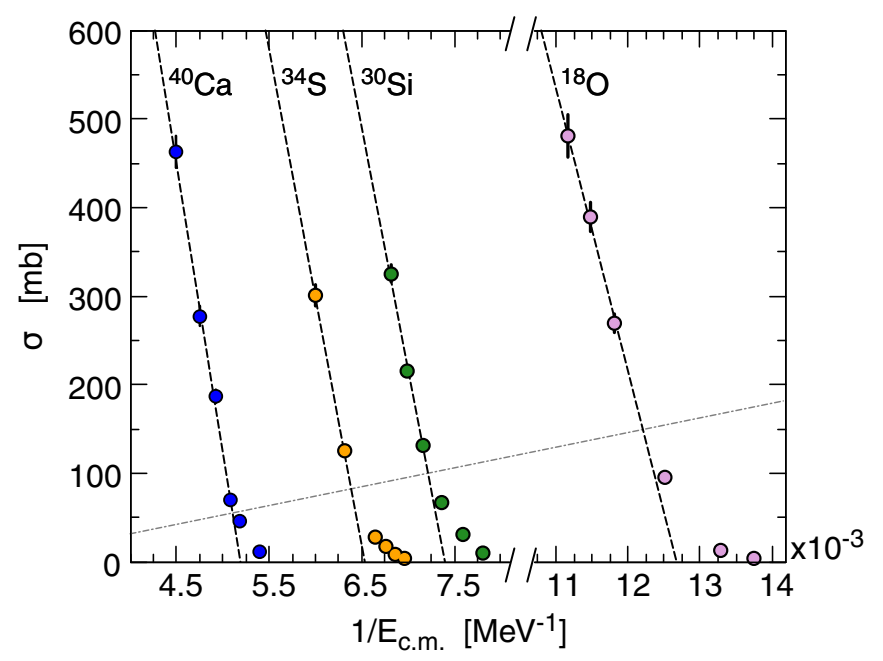

FIG. 9. Experimental capture cross sections as a function of $1 / E_{\mathrm{c} . \mathrm{m}}$. The dashed lines are linear fits using capture cross sections that lie above the dot-dashed line. The inverse of the $x$ intercept is the capture barrier within a classical barrier-passing model. data extending from above the mean barrier energy (to minimize channel coupling effects) up to $50 \%$ above the barrier energy [36]. The fitted region is above the dot-dashed line in Fig. 9. Uncertainties in the experimental barrier energies were evaluated from the statistical uncertainties in the cross sections. Table II gives experimentally determined capture barrier energies and those predicted by the work of Swiatecki et al. [37]. It also gives mean barrier energies predicted from the empirical equation (in $\mathrm{MeV}$ ):

$$
V_{B, \text { c.m. }}=\left[0.0585 \ln \left(Z_{1} Z_{2}\right)+0.5785\right] \frac{Z_{1} Z_{2}}{A_{1}^{1 / 3}+A_{2}^{1 / 3}}
$$

TABLE II. Comparisons of experimental capture barriers with capture barriers with systematics from Eq. (2) and Ref. [37].

\begin{tabular}{lccr}
\hline \hline & \multicolumn{3}{c}{ Fusion (capture) barriers (MeV) } \\
\cline { 2 - 4 } System & Experiment & Empirical Eq. (2) & Ref. [37] \\
\hline${ }^{18} \mathrm{O}+{ }^{232} \mathrm{Th}$ & $78.9 \pm 0.5$ & 79.1 & 78.0 \\
${ }^{30} \mathrm{Si}+{ }^{232} \mathrm{Th}$ & $135.2 \pm 0.7$ & 135.6 & 135.7 \\
${ }^{34} \mathrm{~S}+{ }^{232} \mathrm{Th}$ & $153.1 \pm 0.6$ & 153.9 & 154.8 \\
${ }^{40} \mathrm{Ca}+{ }^{232} \mathrm{Th}$ & $192.8 \pm 0.5$ & 191.2 & 193.8 \\
\hline \hline
\end{tabular}




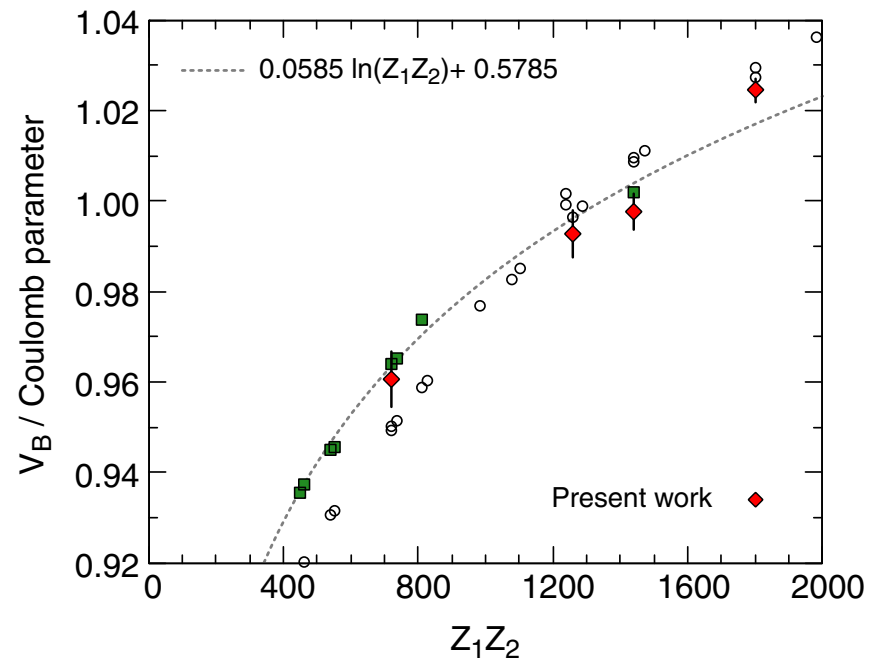

FIG. 10. A plot of the capture (fusion) barrier normalized by the Coulomb parameter $\left[Z_{1} Z_{2} /\left(A_{1}^{1 / 3}+A_{2}^{1 / 3}\right)\right]$ as a function of the charge product. The dashed line is the barrier predicted by Eq. (2) that is an empirical fit to the previous published experimental capture barrier energies (green squares) [3,20,21,38-40]. Hollow circles are predicted barriers for 21 reactions deduced from Ref. [37]. Red diamonds are experimental capture barriers determined in this work (see Fig. 9).

where 1 and 2 refer the two colliding nuclei. This relationship was determined by fitting previous published experimental capture barrier energies in this mass region [3,20,21,38-40], as shown in Fig. 10.

\section{Coupled-channels calculations of capture}

The experimental capture barrier energies were used to constrain CC calculations of capture cross sections. As well as providing predicted capture cross sections, the calculations provided angular momentum distributions for the TSM model calculations used to fit the fission angular distribution for ${ }^{18} \mathrm{O}+{ }^{232} \mathrm{Th}$ and ${ }^{30} \mathrm{Si}+{ }^{232} \mathrm{Th}$, and for the MAD simulations used to extrapolate the angular distributions for ${ }^{34} \mathrm{~S}+{ }^{232} \mathrm{Th}$ and ${ }^{40} \mathrm{Ca}+{ }^{232} \mathrm{Th}$ to angles close to the beam axis.

The CC calculations of capture were carried out using CCFULL $[4,45]$. Calculated capture cross sections $\left(\sigma_{c c}\right)$ depend very sensitively at and below the average barrier energy on details of the coupling included in the CC calculations $[3,46]$. However, in this work it is the experimental capture cross sections $\left(\sigma_{\text {cap }}\right)$ at energies above the barrier that are of interest. Here the smaller effects of couplings that result in potential (and thus barrier energy) renormalization were accounted for as described below.

The CC calculations used a deep Woods-Saxon nuclear potential, with the depth scaling with $Z_{1} Z_{2}$, to avoid coupling effects causing the disappearance of the potential pocket at the higher angular momenta. A fixed diffuseness parameter $a_{0}=0.65 \mathrm{fm}$ was used, which is a typical value reproducing elastic and quasielastic scattering data at both sub-barrier and above-barrier energies [47-49]. Standard collective (vibrational and rotational) couplings in the projectile and target nuclei were included, as shown in Table III. To match the
TABLE III. The spin and parity $\left(J^{\pi}\right)$, excitation energy $\left(E_{x}\right)$, type of coupling (rotational or vibrational), multipolarity $(\lambda)$, and deformation parameters $\left(\beta_{\lambda}\right)$ used in the CC calculations for the target and projectile nuclei [41-43]. The transition strength $B(E \lambda)$ given for ${ }^{40} \mathrm{Ca}$ are taken from the ENSDF [44].

\begin{tabular}{ccccccc}
\hline \hline Nucleus & Coup. & $J^{\pi}$ & $E_{x}(\mathrm{MeV})$ & $\lambda$ & $\beta_{\lambda}$ & $B(E \lambda)$ \\
\hline \multirow{2}{*}{${ }^{232} \mathrm{Th}$} & Rot. & & & 2 & 0.207 & \\
& Rot. & & & 4 & 0.108 & \\
& Vib. & $3^{-}$ & 0.774 & 3 & 0.085 & \\
\hline${ }^{18} \mathrm{O}$ & inert & & & & & \\
${ }^{30} \mathrm{Si}$ & Vib. & $2^{+}$ & 2.235 & 2 & 0.315 & \\
${ }^{34} \mathrm{~S}$ & Vib. & $2^{+}$ & 2.127 & 2 & 0.252 & \\
${ }^{40} \mathrm{Ca}$ & Vib. & $3^{-}$ & 3.737 & 3 & 0.411 & 31 \\
\hline \hline
\end{tabular}

experimental barrier energies, and thus empirically account for the potential renormalization effect of couplings on abovebarrier cross sections, the Woods-Saxon radius parameter was treated as a free parameter. It was chosen such that the mean barrier energy for the calculation (determined in the same way as for experimental cross section data) reproduced the experimental mean capture barrier energy as given in Table II. The CC calculations for each reaction are shown in Fig. 11 by the black lines.

\section{Comparison of FMT fission and calculated capture cross sections}

The comparison of the measured FMT fission (capture) cross sections in Fig. 11 shows that for each reaction, at all energies above the respective capture barriers, the experimental cross sections lie below the $\mathrm{CC}$ calculations. As found in Refs. [5,8], the CC calculations match experiment very well if multiplied by an energy-independent factor $S_{\text {cap }}$ for each reaction, as shown by the red dashed lines.

The extracted values of $S_{\text {cap }}$ are compared with the systematics presented by Newton et al. [5,8] in Fig. 12. The current values of $S_{\text {cap }}$, shown by the red diamonds, agree well with the systematic trend of rapidly decreasing $S_{\text {cap }}$, and thus increasing suppression of capture, with increasing $Z_{1} Z_{2}$. Uncertainties in $S_{\text {cap }}$ resulted mainly from the experimental capture barrier energy uncertainties.

Having obtained agreement with the results of Refs. [5,8], the next step is to test the suggestion made there that deep inelastic scattering (energy dissipation) might be responsible for the increasing suppression of capture cross sections with $Z_{1} Z_{2}$. This is achieved by an empirical (model independent) analysis of the capture-fission and sequential fission cross sections.

\section{E. Sequential fission}

Significant contributions from sequential fission of targetlike nuclei are observed in all these reactions with ${ }^{232} \mathrm{Th}$, shown in Figs. 2 and 3. It must be borne in mind that not all dissipative reactions will result in fission. For pickup reactions, it can be estimated that the fission probability will typically lie in the range 0.5 to 0.9 as the excitation energy increases from the fission threshold to $20 \mathrm{MeV}$ [50]. The 


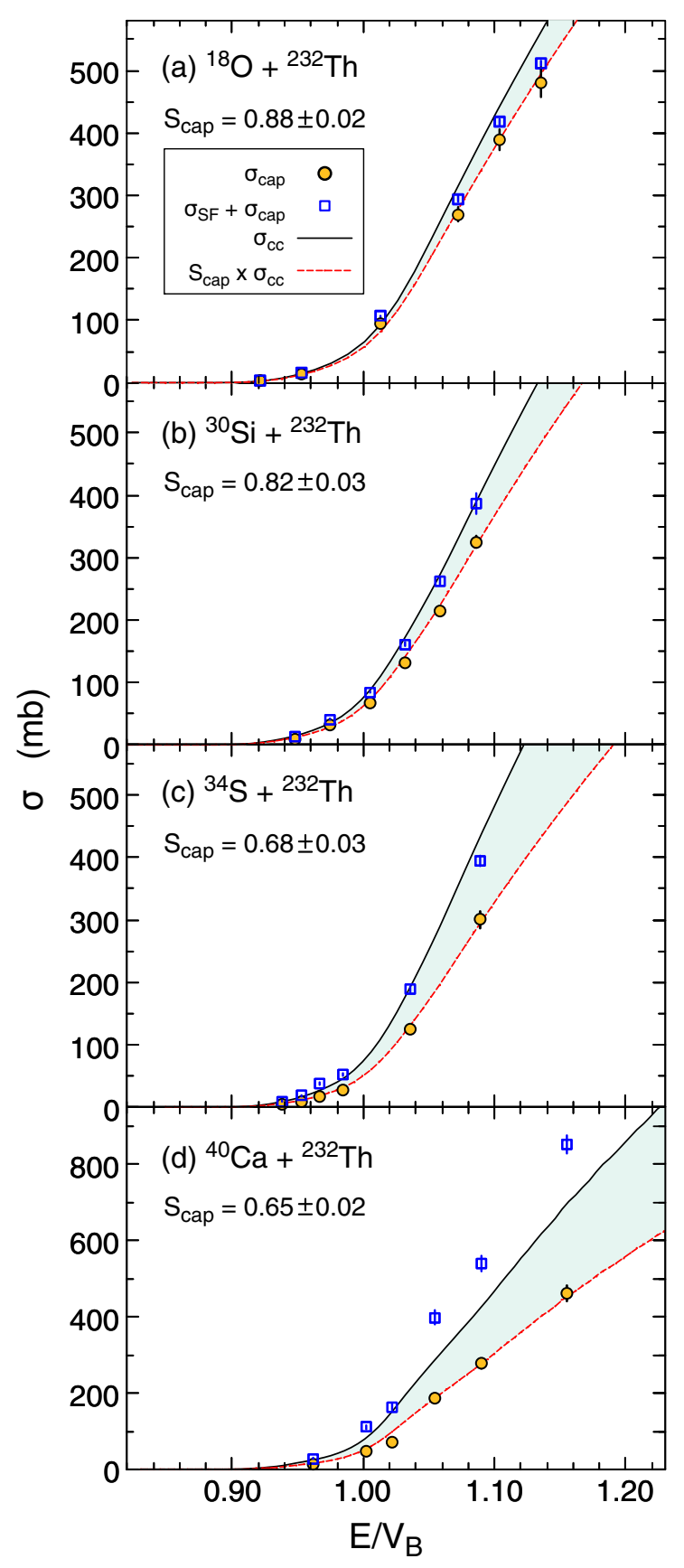

FIG. 11. Experimental FMT capture cross sections (full circles) for ${ }^{18} \mathrm{O},{ }^{30} \mathrm{Si},{ }^{34} \mathrm{~S}$, and ${ }^{40} \mathrm{Ca}+{ }^{232} \mathrm{Th}$. All coupled-channels $(\mathrm{CC}) \mathrm{cal}-$ culations (black lines) used a Woods-Saxon nuclear potential with a diffuseness parameter of $0.65 \mathrm{fm}$. CC calculations scaled by the indicated suppression factor ( $S_{\text {cap }}$, red dashed lines) match the measured cross sections. Hollow squares are the total fission cross sections, which are the sum of the sequential fission (SF) and FMT fission cross sections.

consequences of this will be discussed in more detail in the later interpretation of the sequential fission results.

Determination of the sequential fission cross sections require two issues to be addressed. The first relates to the kinematic coincidence method used in this work. This requires

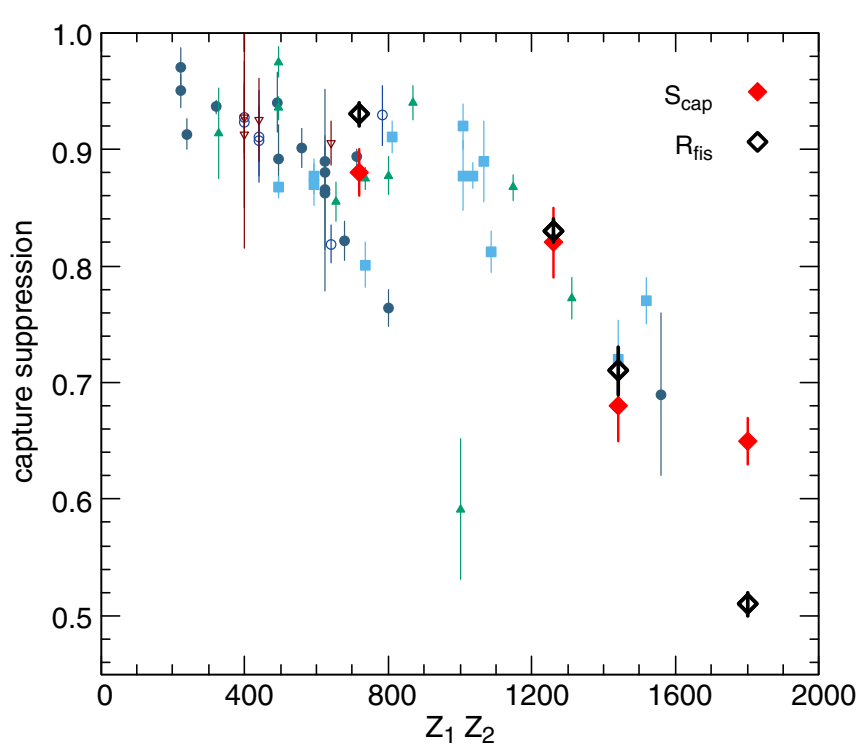

FIG. 12. Capture suppression factor $\left(S_{\text {cap }}\right.$ or $\left.R_{\text {fis }}\right)$ as a function of $Z_{1} Z_{2}$. The results of the current work were overlaid on the top of the results obtained in Refs. [5,8]. Their symbols are described in Ref. [8]. Red solid diamonds $\left(S_{\text {cap }}\right)$ are ratios of the experimental capture cross sections $\left(\sigma_{\text {cap }}\right)$ for ${ }^{18} \mathrm{O},{ }^{30} \mathrm{Si},{ }^{34} \mathrm{~S}$, and ${ }^{40} \mathrm{Ca}+{ }^{232} \mathrm{Th}$ reactions at above-barrier energies to those of $\mathrm{CC}$ calculations $\left(\sigma_{c c}\right)$ using a standard nuclear potential diffuseness $\left(a_{0}=0.65 \mathrm{fm}\right)$. Black hollow diamonds are $R_{\mathrm{fis}}$ evaluated at $E / V_{B} \approx 1.09$ as shown in Fig. 15.

the detection of both fission fragments to obtain information on their origin. The perturbation of the laboratory frame fission kinematics by the ejection of a third (beam-like) particle requires careful selection of the detector angular range, otherwise the coincidence efficiency will be lower for sequential fission than for FMT fission. This selection is described below. The second issue is a consequence of the first. Because of the limited angular range for full-efficiency detection of sequential fission in reactions with the heavier projectiles, the angular distribution of the sequential fission in the laboratory frame cannot be determined over a wide angular range. Both issues have been addressed, and are described below. Thus firm conclusions have been obtained on the relationship between suppression of capture cross sections and energy dissipation.

\section{Detection efficiency for sequential fission}

The CUBE detectors were configured to optimize the geometrical efficiency to detect coincident FMT fission fragments, based on the expected angles between fission fragment velocity vectors, namely the scattering folding angle $\left(\theta_{12}\right)$ and the azimuthal folding angle $\left(\phi_{12}=180^{\circ}\right)$. Despite the large angular coverage of the MWPC detectors, for sequential fission (unlike FMT fission [51]) not all fragments detected in the backward angle MWPC detector will have the complementary fragment detected at the forward angle. Thus the efficiency of detection of sequential fission events can be smaller than for FMT fission. The broader distribution in both $\theta_{12}$ and $\phi_{12}$ for sequential fission events is the result of the distribution of recoil velocities of the nuclei undergoing sequential fission. As seen in Figs. 2 and 3, for the sequential 


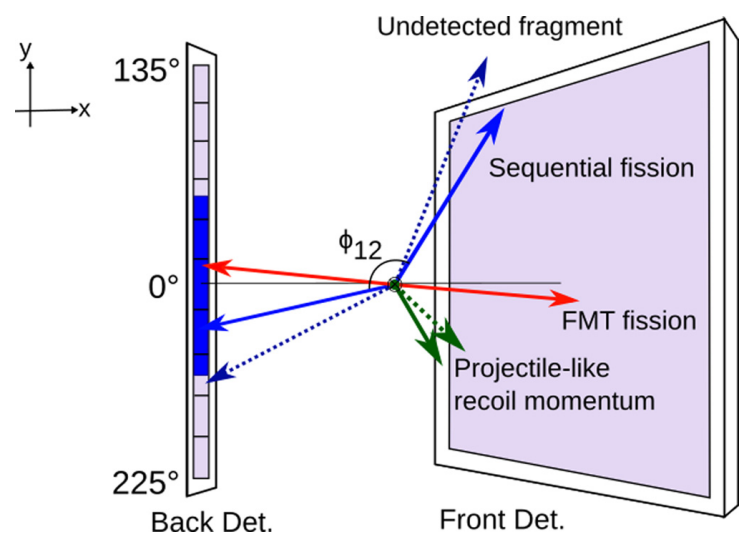

FIG. 13. Schematic view of azimuthal folding angle $\phi_{12}$ of fission fragments with respect to the MWPCs arrangement. There is a higher chance of undetected fission fragment pairs (blue dotted lines) for sequential fission following transfer reactions due to the probability of large $v_{\text {perp }}$ (green arrows) compared to FMT fission (red lines). A certain angular range (blue area) in the back detector allows detection of both FMT and sequential fission with the same efficiency (full lines).

fission events, as the projectile becomes heavier and more energetic more momentum is transferred to the target-like nucleus, leading to larger recoil velocities and thus a wider spread in sequential fission folding angles. An increasing fraction of sequential fission events will thus not be registered, as sketched in Fig. 13 by the dotted arrows. To avoid many assumptions that would have to be made to simulate the detection efficiency for sequential fission events, a limited angular range was chosen in the backward angle MWPC such that, for sequential fission events, the complementary fragment would be detected in the forward angle MWPC with full efficiency.

These angular ranges for each reaction were determined by comparing the ratio of the number of sequential fission to FMT fission events as a function of the angle bin in the back detector. For example, the $\phi$ angular coverage for a fixed $\theta$ range in the back detector was divided into intervals of $10^{\circ}$ as shown in Fig. 14. Each slice had the same gate size $(\Delta \theta \times \Delta \phi)$. If there were no efficiency drops as $\phi$ varies, the ratio should be constant. For gates closer to the detector edges, the ratio is lower, indicating the loss of coincidence detection efficiency. As expected both FMT fission and sequential fission were detected with full coincidence efficiency in the middle of the back detector, as indicated by the pink shaded band in Fig. 14(b). This search procedure was performed for each beam and energy to determine systematically the appropriate angular ranges to obtain reliably the sequential fission to FMT fission ratios.

\section{Ratio of sequential fission to total fission yields}

The first step to investigate the relationship between sequential fission and capture cross sections was the determination of the ratio of the yields of FMT fission to (sequential fission + FMT fission).

Within the angular range selected to give full coincidence efficiency for both sequential fission and FMT fission (capture), the numbers of FMT fission $N_{\mathrm{FMT}}$ and of sequential
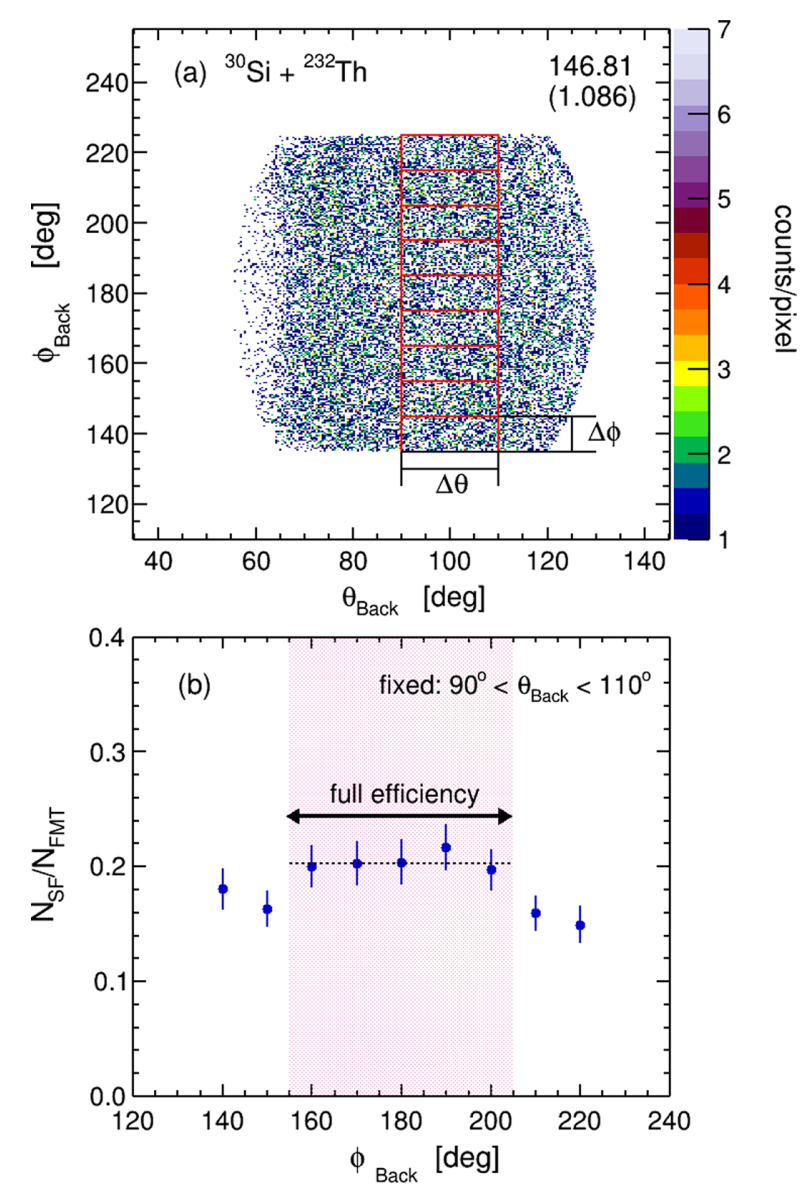

FIG. 14. Panel (a) shows detector coverage in laboratory angle $\theta$ and azimuthal angle $\phi$ for the backward angle detector (back detector). Red squares $(\Delta \theta \times \Delta \phi)$ are gates for efficiency testing (see text). (b) Ratio of sequential fission $\left(N_{\mathrm{SF}}\right)$ to FMT fission $\left(N_{\mathrm{FMT}}\right)$ events observed in given angular ranges (red squares) for ${ }^{30} \mathrm{Si}+{ }^{232} \mathrm{Th}$ at $E_{\mathrm{c} . \mathrm{m} .}=146.81 \mathrm{MeV}$. The shaded band is considered as a relatively constant ratio range, regarding as a full conscience efficiency.

fission $N_{\mathrm{SF}}$ were extracted from the corresponding velocity scatter plots (such as shown for the full angular range in Figs. 2 and 3). These are presented in Fig. 15 in terms of the ratio $R_{\text {fis }}$ of $N_{\text {FMT }}$ to total fission events $\left(N_{\text {Tot }}\right)$ as a function of $E / V_{B}$. The ratio shows clear systematic trends as a function of $Z_{1} Z_{2}$ and $E / V_{B}$, giving confidence in the approach used, and also providing key insights into the physical processes occurring.

These insights are obtained from the observed energy dependence of $R_{\mathrm{fis}}$ and the knowledge that the FMT fission, resulting from capture, falls rapidly below the barrier due to quantum tunneling. The rapid fall in $R_{\text {fis }}$ below the barrier seen in Fig. 15 can therefore only occur if $N_{\mathrm{SF}}$ falls less rapidly than $N_{\mathrm{FMT}}$ at energies below the barrier. This means that, even at distances larger than the capture barrier radius, target-like nuclei are populated at excitation energies above the fission barrier (leading to sequential fission). Well above the barrier, $R_{\mathrm{fis}}$ increases more slowly, perhaps approaching a saturation value. 


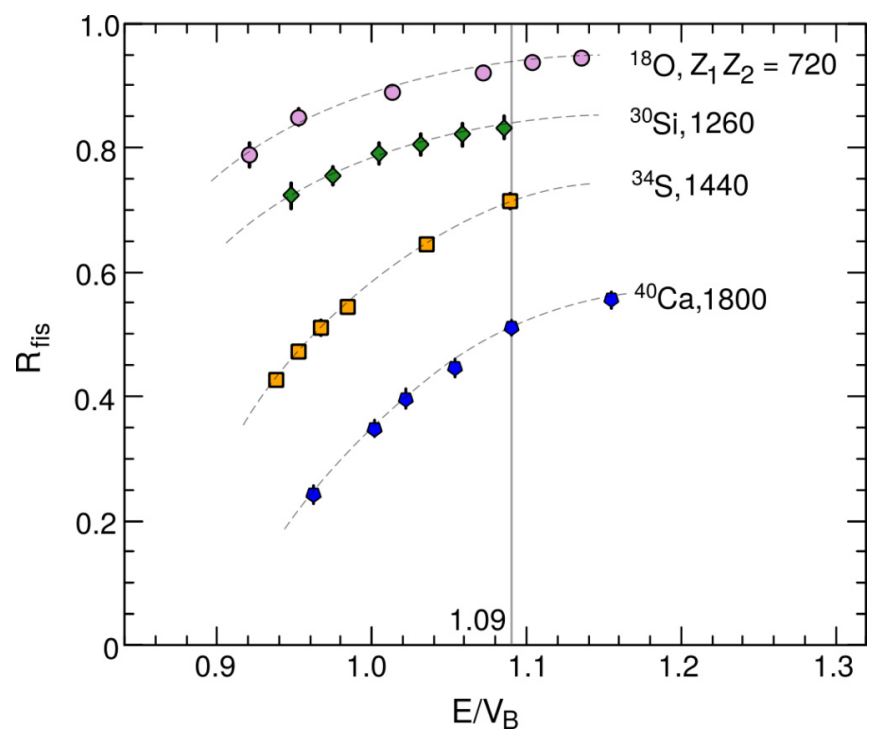

FIG. 15. The ratio $R_{\text {fis }}$ of FMT fission $\left(N_{\mathrm{FMT}}\right)$ to the total fission $\left(N_{\mathrm{Tot}}\right)$ as a function of energy normalized to the capture barriers for ${ }^{18} \mathrm{O},{ }^{30} \mathrm{Si},{ }^{34} \mathrm{~S}$, and ${ }^{40} \mathrm{Ca}+{ }^{232} \mathrm{Th}$ reactions. As $Z_{1} Z_{2}$ increases at a given $E / V_{B}$, the fraction of sequential fission events increases. The curves guide the eye to show overall trends. At $E / V_{B}=1.09$, the ratio of FMT fission to the total fission $\left(R_{\mathrm{fis}}\right)$ was estimated (see text for details).

Thus the observed experimental dependence of $R_{\mathrm{fis}}$ on $E / V_{B}$ suggests qualitatively that the sequential fission events can arise from internuclear separations outside the capture barrier. Analysis of the total fission cross sections (see Sec. III E 3) allows a more quantitative conclusion about this question.

The values of $R_{\mathrm{fis}}$ at a given $E / V_{B}$ in Fig. 15 also show a rapid decrease with increasing $Z_{1} Z_{2}$, indicating the increasing probability of sequential fission. At $E / V_{B}=1.09$ (indicated by the vertical line in Fig. 15), the values of $R_{\mathrm{fis}}$ fall from 0.93 for ${ }^{18} \mathrm{O}$ to 0.51 for ${ }^{40} \mathrm{Ca}$. This decrease is likely to result from increasing probabilities of (multi)nucleon transfer reactions. This might be expected as a result of the increasing matter density overlap required to counter the increasing Coulomb repulsion at the capture barrier radius with higher $Z_{1} Z_{2}$. This is discussed in detail in Sec. IV.

The values $R_{\mathrm{fis}}$ are shown in Fig. 12, where it can be seen that they are correlated strongly with $S_{\text {cap }}$, both depending on $Z_{1} Z_{2}$. For the lighter projectile $\left({ }^{18} \mathrm{O}\right), R_{\mathrm{fis}}$ is slightly larger than $S_{\text {cap }}$. For the intermediate mass projectile $\left({ }^{30} \mathrm{Si}\right.$ and $\left.{ }^{34} \mathrm{~S}\right), R_{\mathrm{fis}}$ is close to $S_{\text {cap }}$. For the ${ }^{40}$ Ca projectile reactions, $R_{\text {fis }}$ is smaller than $S_{\text {cap }}$.

The remaining question is whether the cross sections for the energy dissipative transfer reactions (deep inelastic scattering) can give clearer insight than the ratios presented in Fig. 15. To answer this, the total cross sections for sequential fission were determined, and compared with those for FMT fission and from CC model calculations of capture, as described below.

\section{Cross section of sequential fission}

As described in Sec. III E 1 the angular distribution of the sequential fission events could not be determined because of the broad spread in correlation angles of the two fragments. However their differential cross section $d \sigma / d \Omega\left(\left\langle\theta_{\text {c.m. }}\right\rangle\right)$ could be obtained at the mean angle $\left\langle\theta_{\text {c.m. }}\right\rangle$ of the range selected to give full coincidence efficiency. This was determined by multiplying the deduced FMT fission differential cross section by the observed ratio of sequential fission to FMT fission counts in that angular range.

The differential cross sections $d \sigma / d \Omega$ for sequential fission were translated to total cross section under two different assumptions about the angular distribution of sequential fission. The first was that the angular distributions follow a $1 / \sin \theta_{\text {c.m. }}$ distribution. The second was to assume that the sequential fission events are distributed isotropically in the reaction c.m. frame. In this case, the total sequential fission cross section is $2 \pi d \sigma / d \Omega\left(\left\langle\theta_{\text {c.m. }}\right\rangle\right)$.

Determination of the total cross section of an isotropic angular distribution and a $1 / \sin \theta_{\text {c.m. }}$ distribution from a measurement of $d \sigma / d \Omega$ at a single angle gives the same result if $d \sigma / d \Omega$ is measured at $\theta_{\text {c.m. }}=140.5^{\circ}$. Since the mean c.m. angle of the laboratory angular range selected to give full sequential fission efficiency was typically $\theta_{\text {c.m. }} \approx 130^{\circ}$, the extracted total sequential fission cross section is rather insensitive to the assumed shape of the angular distribution. The final sequential fission cross sections were taken as the average of those determined from the two assumed angular distributions. The uncertainties were taken to extend from the average to these limiting values, and were added in quadrature with statistical uncertainties to give the final experimental uncertainty. Results are shown in Fig. 11.

\section{INTERPRETATION OF SEQUENTIAL AND TOTAL FISSION CROSS SECTIONS}

To explain the systematic suppression of capture cross sections at above-barrier energies, it was suggested $[5,8]$ that energy dissipation before reaching the capture barrier could be causing the system to be reflected from the barrier, rather than passing over it. If the observed sequential fission cross sections were equal to this dissipative cross section, the sum of FMT fission and sequential fission cross sections should match the capture cross sections calculated using a model without inclusion of energy dissipation outside the capture barrier (e.g., the coupled-channels model).

\section{A. Total fission cross sections}

Summing the cross sections of FMT fission and sequential fission gives the total fission cross section:

$$
\sigma_{\mathrm{fis}}=\sigma_{\mathrm{FMT}}+\sigma_{\mathrm{SF}}
$$

These are shown for each reaction by the open blue squares in Fig. 11. Overall, the agreement of $\sigma_{\text {fis }}$ with the CC capture calculations is better than $\sigma_{\text {cap }}$. This gives general support to the idea that capture at above-barrier energies is suppressed by energy dissipation. However, at the highest energies, for ${ }^{18} \mathrm{O}+{ }^{232} \mathrm{Th}$ the $\sigma_{\text {fis }}$ lie slightly below the calculations, while for ${ }^{40} \mathrm{Ca}+{ }^{232} \mathrm{Th}$ they lie significantly above, shown in Figs. 11(a) and 11(d), respectively. To investigate possible rea- 
sons for this trend, a more detailed discussion of the different origins of sequential fission is necessary.

\section{B. Origins of sequential fission}

Sequential fission can result from

(1) dissipative transfer reactions on peripheral (grazing) trajectories that could not result in capture;

(2) dissipative transfer reactions on more central trajectories that would have resulted in capture, but capture was prevented by kinetic energy dissipation associated with the transfer process;

(3) quasifission resulting in a very asymmetric mass-split, with subsequent fission of the target-like nucleus.

It is clear from the above list that $\sigma_{\mathrm{SF}}$ in Eq. (3) is likely to have contributions from peripheral trajectories. If all peripheral processes were followed by fission (i.e., the probability of fission $P_{\text {fis }}=1$ ) then $\sigma_{\mathrm{FMT}}+\sigma_{\mathrm{SF}}$ would be equal to the reaction cross section $\left(\sigma_{\mathrm{rec}}\right)$. However, it is not so straightforward because, as discussed in below, the type of SF trigger mechanism (1-3 above) determines the excitation energies that are populated (i.e., how high above the fission barrier). This, in turn, affects the probability of fission and thus the SF cross section. It therefore follows that $\sigma_{\text {cap }}<\left(\sigma_{\mathrm{FMT}}+\sigma_{\mathrm{SF}}\right)<\sigma_{\text {rec }}$.

\section{Sequential fission probabilities}

The probability of sequential fission following a triggering reaction will depend on the height of the fission barrier (related to the mass and charge or fissility) of the heavy nucleus and on the excitation energy. The discussion of probability then is framed in terms of these variables.

In the case of (multi)nucleon transfer processes, positive effective $Q$ values [14] for charge transfer reactions favour projectile stripping, giving target-like nuclei with higher $Z$ (and thus higher fissility) than ${ }^{232} \mathrm{Th}$. These transfer processes can also lead to large kinetic energy loss [52], and correspondingly to high excitation energies. For the lightest projectile investigated here $\left({ }^{18} \mathrm{O}\right)$, excitation energies $E_{x}$ up to $20 \mathrm{MeV}$ [14] are populated (although with low probability) even at sub-barrier energies. It would be expected, and has been demonstrated $[14,52]$, that the mean excitation energy increases rapidly with increasing matter overlap. Thus the heavier the projectile is, the higher the expected $E_{x}$, and correspondingly the higher the fission probability.

For simple single nucleon transfer reactions the average excitation energy, and thus the average fission probability, would be expected to be quite low. Thus the contribution of these processes to the sequential fission cross sections should be small. The low excitation energies also mean that these reactions will have little influence in suppressing fusion. In contrast, the multinucleon transfer reactions, prevalent for the heavier projectiles, lead to higher excitation energies resulting in a large fission probability. They thus give a high probability of providing an experimental (fission) trigger indicating that they occurred, and are also expected to have a large effect on capture cross sections for those trajectories that would otherwise have passed over the capture barrier.
Fission probabilities of $\mathrm{Np}$ isotopes as a function of $E_{x}$ have been determined for reactions of protons with isotopes of uranium by Boyce et al. [50], through careful experiments and analysis. Just above the fission barrier, the fission probabilities ranged from 0.4 to 0.8 , while at excitation energies over $20 \mathrm{MeV}, P_{\text {fis }}$ is around 0.9 for all isotopes of $\mathrm{Np}$. These $\mathrm{Np}$ isotopes may represent quite well the heavy products of multinucleon stripping reactions, thus $P_{\text {fis }}$ might be expected to be in the range 0.8 to 0.9 , because of the expected high mean excitation energies.

\section{Sequential fission following quasifission}

Quasifission typically shows full kinetic energy damping, and thus the highest available excitation energy for a given mass split. However, following equilibration of the charge to mass ratio, the mass flow tends to be towards mass symmetry, so the heavy QF products are lighter and less fissile than the target nucleus. With increasing mass flow towards symmetry, once the mass region around ${ }^{208} \mathrm{~Pb}$ is reached, where the fission barriers are very high, the probability of sequential fission should become negligible at the excitation energies relevant in these reactions.

The mass-ratio distributions evident from the MADs shown in Fig. 5 indicate which reactions might have a contribution from sequential fission associated with quasifission. For the lighter projectiles, the quasifission yield close to the mass of the projectile is zero or small. However, for ${ }^{40} \mathrm{Ca}+{ }^{232} \mathrm{Th}$, the experimental MADs do show a significant contribution from fast mass-asymmetric QF, where the heavy QF fragment may undergo fission. Conceptually this should be part of the experimental capture cross section, having originated from a trajectory passing inside the capture barrier.

Based on the conclusions of Refs. [23,25], this should represent only a small loss of the capture cross section, and in this work it will be included in the sequential fission cross section, so will not be omitted in the subsequent interpretation of the total fission cross sections.

\section{Discussion of total fission cross sections}

For ${ }^{40} \mathrm{Ca}+{ }^{232} \mathrm{Th}$ it is clear in Fig. 11(d) that the total fission cross section is significantly larger than the expected capture cross section. For this reaction the average excitation energies and thus fission probabilities are expected to be higher than for the ${ }^{18} \mathrm{O},{ }^{30} \mathrm{Si}$, and ${ }^{34} \mathrm{~S}$ reactions. Thus sequential fission cross sections will more closely represent the total dissipative (noncapture) cross section. The excess fission yield shows that deep inelastic and multinucleon transfer cross sections must result not only from trajectories that would have led to capture in the absence of energy dissipative processes, but also from more peripheral trajectories that would not have led to capture. It is concluded that the total sequential fission cross section must therefore have significant contributions both from capture and no-capture trajectories.

For the ${ }^{30} \mathrm{Si}$ and ${ }^{34} \mathrm{~S}$ reactions, shown in Figs. 11(b) and 11(c) respectively, the experimental total fission cross sections agree very well with the above-barrier capture calculations. The simplest interpretation is that fissioning deep inelastic events that would have led to capture are responsible for 
the suppression of the capture cross sections. Based on the observation for the ${ }^{40} \mathrm{Ca}+{ }^{232} \mathrm{Th}$ reaction, it seems likely that there are some deep inelastic events on trajectories that would have led to capture that do not result in fission. Because of the matching between $\sigma_{\text {fis }}$ and the CC capture calculations, these are almost compensated by fissioning deep-inelastic events on trajectories that would not have led to capture.

The total fission cross sections for ${ }^{18} \mathrm{O}+{ }^{232} \mathrm{Th}$ lie below the $\mathrm{CC}$ predictions is shown in Fig. 11(a). Transfer reactions with ${ }^{18} \mathrm{O}$ should show a relatively lower fission probability. Thus the small deficit in the total fission cross section is not surprising. It is very likely a result of the fission "signal" for energy dissipation having lower efficiency (probability) in this reaction.

\section{CONCLUSIONS}

This investigation of energy dissipation probes two related but independent experimental observables determined for the same reactions. The first is the capture cross section, and follows the approach of Newton et al. [5,8], evaluating the suppression of capture from the ratio of measured to calculated capture cross sections. The most novel aspect of this work is the second observable, the yield of sequential fission [expected to result mainly from (multi)nucleon transfer reactions] and its relationship to the suppression of capture cross sections. The aim was to use sequential fission to determine the presence of energy dissipation in heavy ion reactions, and make a quantitative investigation of the relationship between energy dissipative processes and suppression of capture cross sections.

FMT fission and sequential fission for energies from below the capture barrier to $\approx 10 \%$ above, for reactions ${ }^{18} \mathrm{O}+{ }^{232} \mathrm{Th}$, ${ }^{30} \mathrm{Si}+{ }^{232} \mathrm{Th},{ }^{34} \mathrm{~S}+{ }^{232} \mathrm{Th}$, and ${ }^{40} \mathrm{Ca}+{ }^{232} \mathrm{Th}$, spanning $Z_{1} Z_{2}$ values from 720 to 1800 have been measured. Since accurate quasifission cross sections were required, a new approach was developed to extrapolate fast quasifission angular distributions to angles close to the beam axis, using a dynamical model of quasifission. This showed that assuming a $1 / \sin \theta_{\text {c.m. distribu- }}$ tion can give errors of of $\approx 20 \%$ in cross section for fast $\mathrm{QF}$ reactions at near-barrier energies.

Capture barrier energies were determined from the experimental capture cross sections, and coupled-channels calculations matching these barrier energies allowed prediction of expected capture cross sections for a standard nuclear potential diffuseness of $0.65 \mathrm{fm}$. The measured FMT capture cross sections were suppressed relative to those predicted, with a suppression factor varying from 0.93 for ${ }^{18} \mathrm{O}+{ }^{232} \mathrm{Th}$ to 0.65 for ${ }^{40} \mathrm{Ca}+{ }^{232} \mathrm{Th}$, in excellent agreement with previous systematics [5]. The ratio of the yields of sequential fission to FMT fission rises rapidly as the beam energy falls below the capture barrier, indicating that sequential fission is largely associated with processes occurring at radii outside the capture barrier. This was confirmed by the evolution of sequential fission cross sections with $Z_{1} Z_{2}$.

For the ${ }^{40} \mathrm{Ca}+{ }^{232} \mathrm{Th}$ reaction, the sum of FMT fission and sequential fission cross sections exceeds the coupled-channels predicted capture cross sections. This is interpreted as show- ing that energy dissipative processes resulting in sequential fission result both from trajectories that would have passed inside the capture barrier and from trajectories outside the barrier.

We conclude that a picture of energy dissipation outside the capture barrier, as the two nuclei approach, is consistent with the systematic reduction in above-barrier capture cross sections with $Z_{1} Z_{2}$. A model is needed that accounts explicitly for energy dissipation processes, while retaining the coherent coupled-channels features that play a major role in all aspects of near-barrier capture.

\section{ACKNOWLEDGMENTS}

The authors are grateful for Dr. N. Lobanov, Dr. T. Kibedi, and all the accelerator staff at the ANU Heavy Ion Accelerator Facility for support in operating the 14UD and LINAC accelerators. This work was supported by Australian Research Council Discovery Grants No. DP170102318, No. DP170102423, No. DP190100256, No. DP200100601, and No. DE140100784.

\section{APPENDIX: SIMULATION OF FAST QUASIFISSION}

In order to determine cross sections for reactions following capture that do not lead to compound nucleus formation but rather to quasifission $(\mathrm{QF})$, where a "memory" of the projectile mass and direction is often retained, a Monte Carlo calculation based on a phenomenological approach $[18,23]$ was used. This approach uses the characteristics of reaction timescales through the correlation between fission fragment masses and their corresponding emission angles, known as the MAD. The calculated MADs that describe the angular distributions of fast QF over the measured range allow extrapolation to angles close to the beam direction. This Appendix shows the Monte Carlo calculations for ${ }^{40} \mathrm{Ca}+{ }^{232} \mathrm{Th}$. Details for the ${ }^{34} \mathrm{~S}+{ }^{232} \mathrm{Th}$ reaction are given in Ref. [28]

The observed emission angle in center-of-mass frame $\left(\theta_{\mathrm{QF}}\right)$ of a projectile-like fission fragment, as described in Ref. [23], is given by

$$
\theta_{\mathrm{QF}}\left(t_{s}\right)=\pi-\left[\Theta_{\mathrm{in}}+\theta_{\mathrm{rot}}\left(t_{s}\right)+\Theta_{\text {out }}\right],
$$

where $\theta_{\text {rot }}\left(t_{s}\right)$ is the rotation angle of the system while joined together, and $\Theta_{\text {in }}$ and $\Theta_{\text {out }}$ are the angles associated with the classical Coulomb trajectories for the incoming and outgoing nuclei. Being a binary process, the target-like fragment is formed at the complementary angle $\left(\pi-\theta_{\mathrm{QF}}\right)$. The rotation angle during the sticking time $\left(t_{s}\right)$ between contact and scission is estimated from the relationship between the angular velocity $\omega$ and the sticking time $t_{s}, \theta_{\text {rot }}=\omega \times t_{s}$. Expressing the angular velocity in terms of $J$, the orbital angular momentum in the collision and $\langle I\rangle$ the average moment of inertia during the sticking time, gives the rotation angle:

$$
\theta_{\text {rot }}\left(t_{s}\right)=\frac{\sqrt{J(J+1)} \hbar}{\langle I\rangle} t_{s} .
$$

The angular momentum of each event $(J)$ was randomly chosen and weighted by the angular momentum distributions obtained through the capture partial wave cross sections 


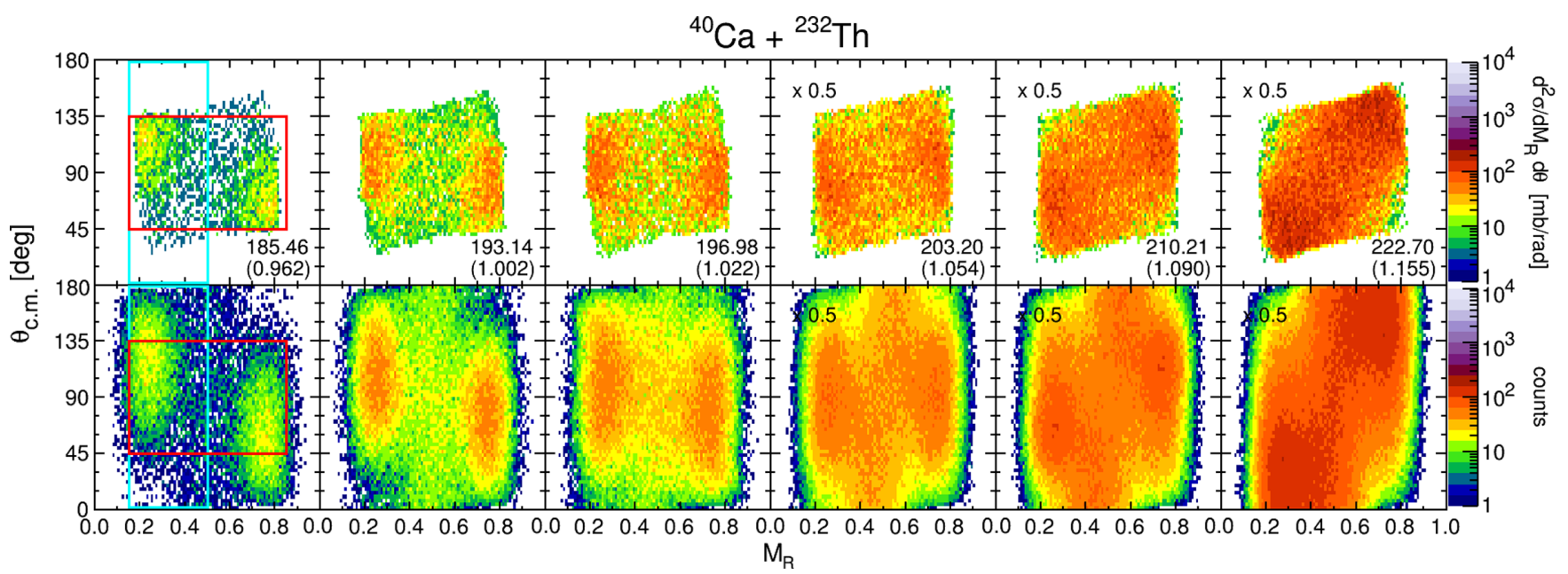

FIG. 16. The upper panels show the experimental solid angle normalized MADs and the lower panels are simulated MADs. Blue and red rectangles are gates used to produce the distributions shown in Figs 17 and $18 . Z$ values of the three highest MADs are scaled by 0.5 .

calculated with the coupled-channels model CCMOD [53]. An average moment of inertia $\langle I\rangle$ was estimated using geometrical relations, assuming a dinuclear (rigid body) after a collision between a spherical projectile and deformed target nuclei.

Based on the work of Ref. [23], the masses of each constituent nucleus were assumed to evolve to reach mass symmetry asymptotically with time. The initial $M_{R}(0)$ is the ratio of projectile mass to the total mass. The mass flow between the two constituents can be defined in terms of the mass ratio $M_{R}$ as follows:

$$
M_{R}\left(t_{s}\right)=\left[M_{R}(0)-0.5\right] \exp ^{-t_{s} / t_{m}}+0.5,
$$

where $t_{s}$ is the sticking time and $t_{m}$ is the mass equilibrium time constant, determined experimentally to be $5.2 \times 10^{-21} \mathrm{~s}$ [23]. This value later confirmed by fully microscopic calculations [54]. The mass ratio of the target-like nuclei is $\left(1-M_{R}\right)$.
The sticking time $\left(t_{s}\right)$ was parametrized as a distribution rather than a single value. The assumed general form of the sticking time distribution was a half-Gaussian followed by an exponential tail. $t_{s}$ is a key parameter to reproduce the simulated MADs since it determines the rotational angle and the amount of mass flow during the evolution of the dinuclear system.

Thorium is a well-deformed prolate nucleus. Different sticking time distributions for tip and side collisions, which are divided at a critical angle $\left(\theta_{\text {crit }}=25^{\circ}\right.$ for ${ }^{34} \mathrm{~S}+{ }^{232} \mathrm{Th}$ and $\theta_{\text {crit }}=30^{\circ}$ for ${ }^{40} \mathrm{Ca}+{ }^{232} \mathrm{Th}$ ), were used. This is because the effect of the contact configuration on the reaction dynamics leads to different QF outcomes [30]. Sticking time distributions were systematically parametrized based on two fundamental observed characteristics: (1) that the QF timescale of tip collisions is faster than that of side collisions and (2) that fast QF leads to asymmetric mass distributions whereas slow QF leads to mass symmetric distributions.

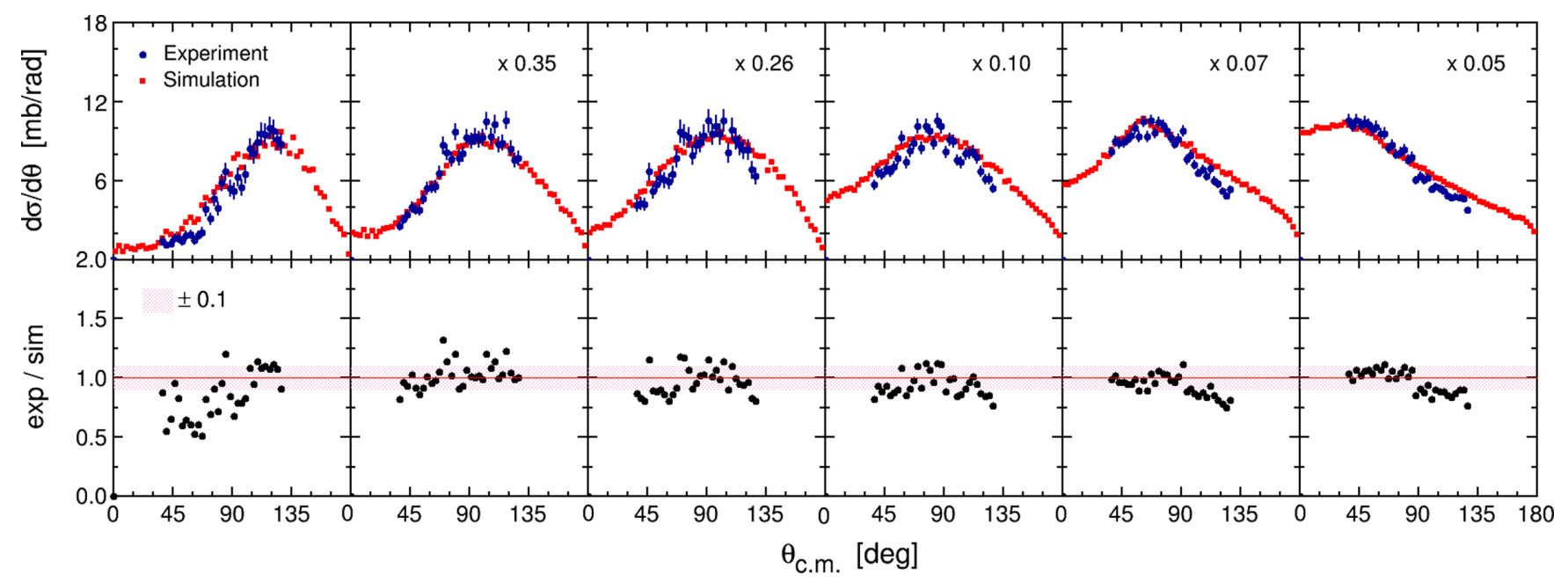

FIG. 17. The upper panels show experimental angular distributions (blue) and simulated distributions (red). The ratios of experiment to simulation are shown in the bottom panels. Perfect agreement $($ ratio $=1)$ is indicated by the red line. The shaded band corresponds to a \pm 0.1 range. 


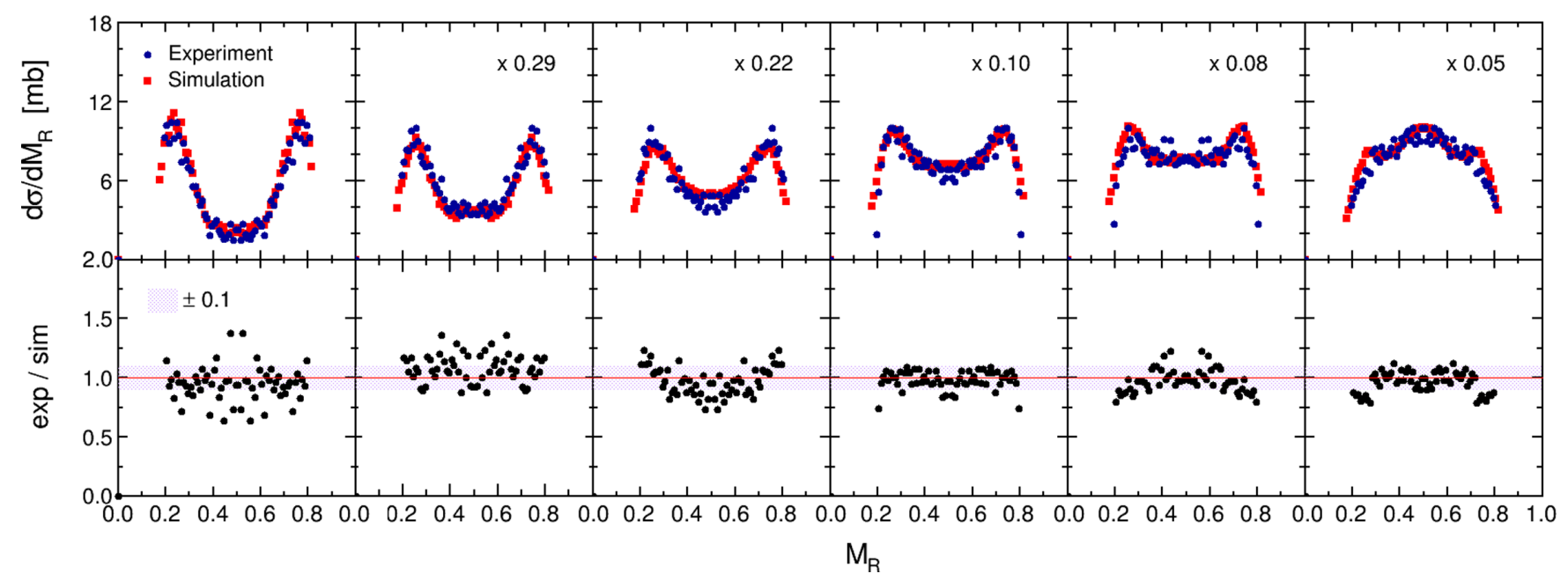

FIG. 18. The upper panels show experimental $M_{R}$ distributions (blue) and simulated distributions (red). The ratios of experiment to simulation are shown in the bottom panels. The shaded band corresponds to a \pm 0.1 range.

A further variable was needed to describe massasymmetric fast QF. As described in Ref. [28], the fast QF was taken to result in a mass split centered near heavy fragment mass close to $\mathrm{Pb}$ isotopes. Impact of the ${ }^{208} \mathrm{~Pb}$ doubly closed shell of QF was indeed demonstrated in Refs. $[55,56]$. Other deformed shell effects, not accounted here, may also affect the outcome of QF reactions [57]. For ${ }^{40} \mathrm{Ca}+{ }^{232} \mathrm{Th}$, the side collisions also required a fraction of mass-asymmetric fast $\mathrm{QF}$ to reproduce the experimental mass ratio distributions at the three highest beam energies. This does not affect the simulated angular distributions.

The top row of panels in Fig. 16 show the measured MADs, with the simulated MADs on the bottom row. Projections on the $x$ axis (applying the red rectangular gate) and the $y$ axis (applying a cyan rectangular gate) for these figures give the angular distributions and $M_{R}$ distributions shown in Figs. 17 and 18 respectively. The top panels of both figures show comparisons between measured distributions (blue points) and simulations (red points). The bottom panels show the ratios of the experimental distributions to the simulated distributions. The simulation followed an iterative process until good agreement was achieved between the experimental data and the simulated data in the region of the experimental angular coverage with full detection efficiency. This resulted in the ratios of measured data and simulated data being close to 1 . There were statistical fluctuations of 5\%-10\%. Overall the simulated MADs successfully reproduced the experimental MADs. However, as beam energy increases, the simulated data are higher than the measured data at backward angles from $100^{\circ}$ to $135^{\circ}$, since the simulations were focused on matching the experimental data with highest yield at more forward angles. This required rescaling the simulated data at the missing angles of angular distributions, as indicated in Fig. 8. This deviation may be because of some correlations not yet taken into account in this simulation. In later work, this issue will be investigated in more detail, through dedicated high statistics measurements.
[1] C. R. Morton, M. Dasgupta, D. J. Hinde, J. R. Leigh, R. C. Lemmon, J. P. Lestone, J. C. Mein, J. O. Newton, H. Timmers, N. Rowley, and A. T. Kruppa, Phys. Rev. Lett. 72, 4074 (1994).

[2] A. B. Balantekin and N. Takigawa, Rev. Mod. Phys. 70, 77 (1998).

[3] M. Dasgupta, D. J. Hinde, N. Rowley, and A. M. Stefanini, Annu. Rev. Nucl. Part. Sci. 48, 401 (1998).

[4] K. Hagino and N. Takigawa, Prog. Theor. Phys. 128, 1061 (2012).

[5] J. O. Newton, R. D. Butt, M. Dasgupta, D. J. Hinde, I. Gontchar, C. R. Morton, and K. Hagino, Phys. Lett. B 586, 219 (2004).

[6] C. L. Jiang, K. E. Rehm, R. V. F. Janssens, H. Esbensen, I. Ahmad, B. B. Back, P. Collon, C. N. Davids, J. P. Greene,
D. J. Henderson, G. Mukherjee, R. C. Pardo, M. Paul, T. O. Pennington, D. Seweryniak, S. Sinha, and Z. Zhou, Phys. Rev. Lett. 93, 012701 (2004).

[7] M. Dasgupta, D. J. Hinde, A. Diaz-Torres, B. Bouriquet, C. I. Low, G. J. Milburn, and J. O. Newton, Phys. Rev. Lett. 99, 192701 (2007).

[8] J. O. Newton, R. D. Butt, M. Dasgupta, D. J. Hinde, I. I. Gontchar, C. R. Morton, and K. Hagino, Phys. Rev. C 70, 024605 (2004).

[9] B. B. Back, H. Esbensen, C. L. Jiang, and K. E. Rehm, Rev. Mod. Phys. 86, 317 (2014).

[10] T. Tamura, Rev. Mod. Phys. 37, 679 (1965).

[11] M. Evers, M. Dasgupta, D. J. Hinde, D. H. Luong, R. Rafiei, R. du Rietz, and C. Simenel, Phys. Rev. C 84, 054614 (2011).

[12] V. Volkov, Phys. Rep. 44, 93 (1978). 
[13] D. Bromley, Treatise on Heavy Ion Science (Springer, New York, 1984).

[14] D. C. Rafferty, M. Dasgupta, D. J. Hinde, C. Simenel, E. C. Simpson, E. Williams, I. P. Carter, K. J. Cook, D. H. Luong, S. D. McNeil, K. Ramachandran, K. Vo-Phuoc, and A. Wakhle, Phys. Rev. C 94, 024607 (2016).

[15] G. Pollarolo, EPJ Web Conf. 17, 08002 (2011).

[16] D. J. Hinde, M. Dasgupta, A. Diaz-Torres, and M. Evers, Nucl. Phys. A 834, 117c (2010).

[17] M. L. Yoneama, E. Jacobs, J. Arruda-Neto, B. Bhandari, D. D. Frenne, S. Pommé, K. Persyn, and K. Govaert, Nucl. Phys. A 604, 263 (1996).

[18] R. du Rietz, E. Williams, D. J. Hinde, M. Dasgupta, M. Evers, C. J. Lin, D. H. Luong, C. Simenel, and A. Wakhle, Phys. Rev. C 88, 054618 (2013).

[19] D. Hinde, C. Morton, M. Dasgupta, J. Leigh, J. Mein, and H. Timmers, Nucl. Phys. A 592, 271 (1995).

[20] D. J. Hinde, M. Dasgupta, J. R. Leigh, J. C. Mein, C. R. Morton, J. O. Newton, and H. Timmers, Phys. Rev. C 53, 1290 (1996).

[21] D. J. Hinde, R. du Rietz, M. Dasgupta, R. G. Thomas, and L. R. Gasques, Phys. Rev. Lett. 101, 092701 (2008).

[22] T. Banerjee, D. J. Hinde, D. Y. Jeung, K. Banerjee, M. Dasgupta, A. C. Berriman, L. T. Bezzina, H. M. Albers, C. E. Düllmann, J. Khuyagbaatar, B. Kindler, B. Lommel, E. C. Simpson, C. Sengupta, B. M. A. Swinton-Bland, T. Tanaka, A. Yakushev, K. Eberhardt, C. Mokry, J. Runke, P. ThörlePospiech, and N. Trautmann, Phys. Rev. C 102, 024603 (2020).

[23] J. Tōke, R. Bock, G. Dai, A. Gobbi, S. Gralla, K. Hildenbrand, J. Kuzminski, W. Müller, A. Olmi, H. Stelzer, B. Back, and S. Bjørnholm, Nucl. Phys. A 440, 327 (1985).

[24] W. J. Swiatecki, Phys. Scr. 24, 113 (1981).

[25] W. Q. Shen, J. Albinski, A. Gobbi, S. Gralla, K. D. Hildenbrand, N. Herrmann, J. Kuzminski, W. F. J. Müller, H. Stelzer, J. Tōke, B. B. Back, S. Bjørnholm, and S. P. Sørensen, Phys. Rev. C 36, 115 (1987).

[26] E. Williams, D. J. Hinde, M. Dasgupta, R. du Rietz, I. P. Carter, M. Evers, D. H. Luong, S. D. McNeil, D. C. Rafferty, K. Ramachandran, and A. Wakhle, Phys. Rev. C 88, 034611 (2013).

[27] C. J. Lin, R. du Rietz, D. J. Hinde, M. Dasgupta, R. G. Thomas, M. L. Brown, M. Evers, L. R. Gasques, and M. D. Rodriguez, Phys. Rev. C 85, 014611 (2012).

[28] E. Prasad, A. Wakhle, D. J. Hinde, E. Williams, M. Dasgupta, M. Evers, D. H. Luong, G. Mohanto, C. Simenel, and K. VoPhuoc, Phys. Rev. C 93, 024607 (2016).

[29] R. du Rietz, D. J. Hinde, M. Dasgupta, R. G. Thomas, L. R. Gasques, M. Evers, N. Lobanov, and A. Wakhle, Phys. Rev. Lett. 106, 052701 (2011).

[30] D. J. Hinde, D. Y. Jeung, E. Prasad, A. Wakhle, M. Dasgupta, M. Evers, D. H. Luong, R. du Rietz, C. Simenel, E. C. Simpson, and E. Williams, Phys. Rev. C 97, 024616 (2018).

[31] H. Rossner, J. R. Huizenga, and W. U. Schröder, Phys. Rev. C 33, 560 (1986).

[32] C. R. Morton, D. J. Hinde, J. R. Leigh, J. P. Lestone, M. Dasgupta, J. C. Mein, J. O. Newton, and H. Timmers, Phys. Rev. C 52, 243 (1995).

[33] C. R. Morton, A. C. Berriman, R. D. Butt, M. Dasgupta, A. Godley, D. J. Hinde, and J. O. Newton, Phys. Rev. C 62, 024607 (2000).
[34] K. Lützenkirchen, J. Kratz, G. Wirth, W. Brüchle, K. Sümmerer, R. Lucas, J. Poitou, and C. Grégoire, Nucl. Phys. A 452, 351 (1986).

[35] V. E. Oberacker, A. S. Umar, and C. Simenel, Phys. Rev. C 90, 054605 (2014).

[36] D. J. Hinde, M. Dasgupta, N. Herrald, R. G. Neilson, J. O. Newton, and M. A. Lane, Phys. Rev. C 75, 054603 (2007).

[37] W. J. Światecki, K. Siwek-Wilczyńska, and J. Wilczyński, Phys. Rev. C 71, 014602 (2005).

[38] J. C. Mein, D. J. Hinde, M. Dasgupta, J. R. Leigh, J. O. Newton, and H. Timmers, Phys. Rev. C 55, R995 (1997).

[39] D. J. Hinde, M. Dasgupta, J. R. Leigh, D. G. Marinaro, and J. O. Newton, Phys. Rev. Lett. 81, 4777 (1998).

[40] J. P. Lestone, A. A. Sonzogni, M. P. Kelly, and R. Vandenbosch, J. Phys. G: Nucl. Part. Phys. 23, 1349 (1997).

[41] S. Raman, C. Nestor, and P. Tikkanen, At. Data. Nucl. Data Tables 78, 1 (2001).

[42] T. Kibédi and R. H. Spear, At. Data. Nucl. Data Tables 80, 35 (2002)

[43] T. Elze and J. Huizenga, Nucl. Phys. A 187, 545 (1972).

[44] Evaluated Nuclear Structure Data File (ENSDF), http://www. nndc.bnl/gov/ensdf.

[45] K. Hagino, N. Rowley, and A. Kruppa, Comput. Phys. Commun. 123, 143 (1999).

[46] R. G. Stokstad, Y. Eisen, S. Kaplanis, D. Pelte, U. Smilansky, and I. Tserruya, Phys. Rev. C 21, 2427 (1980).

[47] W. Reisdorf, J. Phys. G: Nucl. Part. Phys. 20, 1297 (1994).

[48] L. R. Gasques, M. Evers, D. J. Hinde, M. Dasgupta, P. R. S. Gomes, R. M. Anjos, M. L. Brown, M. D. Rodríguez, R. G. Thomas, and K. Hagino, Phys. Rev. C 76, 024612 (2007).

[49] M. Evers, M. Dasgupta, D. J. Hinde, L. R. Gasques, M. L. Brown, R. Rafiei, and R. G. Thomas, Phys. Rev. C 78, 034614 (2008).

[50] J. R. Boyce, T. D. Hayward, R. Bass, H. W. Newson, E. G. Bilpuch, F. O. Purser, and H. W. Schmitt, Phys. Rev. C 10, 231 (1974).

[51] K. Banerjee, D. J. Hinde, M. Dasgupta, E. C. Simpson, D. Y. Jeung, C. Simenel, B. M. A. Swinton-Bland, E. Williams, I. P. Carter, K. J. Cook, H. M. David, C. E. Düllmann, J. Khuyagbaatar, B. Kindler, B. Lommel, E. Prasad, C. Sengupta, J. F. Smith, K. Vo-Phuoc, J. Walshe, and A. Yakushev, Phys. Rev. Lett. 122, 232503 (2019).

[52] L. Corradi, J. Phys. Conf. Ser. 282, 012005 (2011).

[53] M. Dasgupta, A. Navin, Y. Agarwal, C. Baba, H. Jain, M. Jhingan, and A. Roy, Nucl. Phys. A 539, 351 (1992).

[54] C. Simenel, K. Godbey, and A. S. Umar, Phys. Rev. Lett. 124, 212504 (2020).

[55] A. Wakhle, C. Simenel, D. J. Hinde, M. Dasgupta, M. Evers, D. H. Luong, R. du Rietz, and E. Williams, Phys. Rev. Lett. 113, 182502 (2014).

[56] M. Morjean, D. J. Hinde, C. Simenel, D. Y. Jeung, M. Airiau, K. J. Cook, M. Dasgupta, A. Drouart, D. Jacquet, S. Kalkal, C. S. Palshetkar, E. Prasad, D. Rafferty, E. C. Simpson, L. Tassan-Got, K. Vo-Phuoc, and E. Williams, Phys. Rev. Lett. 119, 222502 (2017).

[57] K. Godbey, A. S. Umar, and C. Simenel, Phys. Rev. C 100, 024610 (2019). 